\title{
Phase reduction of limit-torus solutions to partial differential algebraic equations
}

\author{
Yoji Kawamura $\odot^{*}$ \\ Center for Mathematical Science and Advanced Technology, Japan Agency for Marine-Earth Science and Technology, \\ Yokohama 236-0001, Japan
}

(Received 4 July 2019; published 26 November 2019)

\begin{abstract}
A limit-torus solution describes a traveling and oscillating solution. It is characterized by two phase variables, spatial phase and temporal phase, which indicate the position and oscillation of the solution, respectively. Here, we develop a theoretical framework for the phase reduction of limit-torus solutions to partial differential algebraic equations or partial differential equations with constraints. We derive phase sensitivity functions for the two phases; these functions quantify the spatiotemporal phase responses of the solution under weak perturbations applied at each spatial point and at each time. We consider oscillatory thermal convection in a two-dimensional incompressible Navier-Stokes flow system with lateral periodicity as a prototype.
\end{abstract}

DOI: 10.1103/PhysRevResearch.1.033130

\section{INTRODUCTION}

Nature provides a rich variety of rhythmic systems and synchronization phenomena [1-5]. A rhythmic system is typically described by a limit-cycle solution to an ordinary differential equation. Phase reduction of ordinary limit-cycle oscillators is a robust method that has been extensively utilized for analyzing the synchronization properties of the oscillators [1-12]. Subsequently, collective synchronization exhibited by coupled phase oscillators has been widely investigated for globally coupled systems, nonlocally coupled systems, and complex network systems [1-19].

We recently demonstrated a phase reduction method for limit-cycle solutions to the following partial differential equations: (i) nonlinear Fokker-Planck equations representing collective oscillations of globally coupled noisy dynamical elements [20,21], (ii) fluid equations representing oscillatory thermal convection in ordinary Hele-Shaw cells [22,23], (iii) reaction-diffusion equations representing spatiotemporal rhythms in chemical and biological systems [24,25], and (iv) fourth-order nonlinear partial differential equations representing beating flagella [26]; more precisely, the first and second equations are partial differential integral equations. This method can be considered as a generalization of the conventional phase reduction method for ordinary differential equations.

Several rhythmic spatiotemporal patterns can be described by limit-cycle solutions to partial differential equations [27-41]. However, some systems possess additional spatial translational symmetry, and therefore, rhythmic spatiotemporal patterns in the systems cannot be described by limit-cycle

\footnotetext{
*ykawamura@jamstec.go.jp

Published by the American Physical Society under the terms of the Creative Commons Attribution 4.0 International license. Further distribution of this work must maintain attribution to the author(s) and the published article's title, journal citation, and DOI.
}

solutions. For example, annuli and spheres possess continuous rotational symmetry, namely, continuous translational symmetry in the azimuthal direction [36-42]. The presence of spatiotemporal rhythms in these systems causes the emergence of two phase modes: spatial phase and temporal phase. Such spatiotemporal rhythms are described by limit-torus solutions. The synchronization of spatiotemporal rhythms with the two phase modes has been experimentally investigated [43-45] in systems of rotating fluid annuli that exhibit rotating and oscillating convection (i.e., amplitude vacillation [40-42]), which is an analog of atmospheric circulation. Hence, in a recent study [46], we developed a generalized phase reduction method for limit-torus solutions to partial differential equations. Here, we considered oscillatory thermal convection in a cylindrical Hele-Shaw cell that is laterally periodic. Owing to its cylindrical shape, this cell exhibits spatial translational symmetry in the lateral direction. The oscillatory thermal convection was therefore described by a limit-torus solution, which possessed both spatial and temporal phases.

However, rotating and oscillating convection in a system of rotating fluid annulus is described by an incompressible fluid equation. This equation can be considered as a partial differential algebraic equation because there is no dynamical equation for the pressure but only an incompressible condition. Therefore, a phase reduction method for limit-torus solutions to partial differential algebraic equations is highly desirable. This is the objective of the present study. As a significant extension of the earlier study [46], we consider traveling and oscillating thermal convection in a two-dimensional incompressible Navier-Stokes flow system that is laterally periodic. The dynamics of such a system can be described by a partial differential algebraic equation. Moreover, the traveling and oscillating thermal convection is described by a limit-torus solution that exhibits both spatial and temporal phases. Here, we develop a theoretical framework for the phase reduction of traveling and oscillating thermal convection. This theory can be considered as a generalized phase reduction method for limit-torus solutions to partial differential algebraic equations. Further, this method enables us to describe the dynamics 
of the thermal convection by two phases (i.e., spatial and temporal phases) and facilitates the theoretical analysis of spatiotemporal phase synchronization properties of the thermal convection in weakly coupled systems.

This paper is organized as follows. In Sec. II with Appendix A, we formulate a theory for the phase reduction of traveling and oscillating thermal convection. In Sec. III with Appendixes B and C, we illustrate the theory using a numerical analysis of the thermal convection. Concluding remarks are given in Sec. IV.

\section{PHASE DESCRIPTION OF LIMIT-TORUS SOLUTIONS}

In this section, we present a theoretical framework for the phase description of oscillatory thermal convection in a two-dimensional incompressible Navier-Stokes flow system that is laterally periodic. This theory can be considered as a generalization of our phase description method for oscillatory thermal convection in the Darcy flow system [46].

\section{A. Dimensionless form of incompressible Navier-Stokes equations}

The two-dimensional incompressible Navier-Stokes equation under the Boussinesq approximation can be expressed as follows $[27,28,38,39]$ :

$$
\begin{gathered}
0=\nabla \cdot \boldsymbol{v}, \\
\frac{\partial}{\partial t} v(x, y, t)=-\boldsymbol{v} \cdot \nabla v-\frac{\partial p}{\partial x}+\operatorname{Pr} \nabla^{2} v, \\
\frac{\partial}{\partial t} w(x, y, t)=-\boldsymbol{v} \cdot \nabla w-\frac{\partial p}{\partial y}+\operatorname{Pr} \operatorname{Ra} \theta+\operatorname{Pr} \nabla^{2} w, \\
\frac{\partial}{\partial t} \theta(x, y, t)=-\boldsymbol{v} \cdot \nabla \theta+w+\nabla^{2} \theta,
\end{gathered}
$$

where $p, v=(v, w)^{\mathrm{T}}$, and $\theta$ denote the pressure, fluid velocity field, and convective component of the temperature field, respectively. Here, Eqs. (1), (2) and (3), and (4) represent the incompressible condition for the fluid velocity field $\boldsymbol{v}$, Navier-Stokes flow under the Boussinesq approximation, and advection-diffusion process for the convective component of the temperature, respectively. The Prandtl number and Rayleigh number are denoted by Pr and $\mathrm{Ra}$, respectively.

The system is defined in the following region: $x \in[0,2 L)$ and $y \in[-1,+1]$. The boundary conditions of the system are as follows. First, this system is assumed to be laterally periodic, and therefore, it satisfies the $2 L$-periodic boundary condition on $x$, i.e.,

$$
\begin{aligned}
& v(x+2 L, y, t)=v(x, y, t), \\
& w(x+2 L, y, t)=w(x, y, t), \\
& \theta(x+2 L, y, t)=\theta(x, y, t) .
\end{aligned}
$$

Second, this system satisfies the Dirichlet zero boundary condition on $y$, i.e.,

$$
\begin{aligned}
& \left.v(x, y, t)\right|_{y= \pm 1}=0, \\
& \left.w(x, y, t)\right|_{y= \pm 1}=0, \\
& \left.\theta(x, y, t)\right|_{y= \pm 1}=0 .
\end{aligned}
$$

Here, the convective component of the temperature field is defined as

$$
T(x, y, t)=-y+\theta(x, y, t) .
$$

The dynamics of the temperature field $T(x, y, t)$ is described by the following advection-diffusion equation:

$$
\frac{\partial}{\partial t} T(x, y, t)=-\boldsymbol{v} \cdot \nabla T+\nabla^{2} T .
$$

The boundary condition for the temperature field on $y$ is given by

$$
\left.T(x, y, t)\right|_{y= \pm 1}=\mp 1,
$$

where the temperature at the bottom $(y=-1)$ is higher than that at the top $(y=+1)$. Substituting Eq. (11) into Eqs. (12) and (13), we can obtain Eqs. (4) and (10), respectively.

Owing to the existence an incompressible condition represented by Eq. (1), a set of Eqs. (1), (2), (3), and (4) can be considered as a partial differential algebraic equation (see, e.g., Refs. [47,48]). In other words, there is no dynamical equation for the pressure $p(x, y, t)$. The above equations can be expressed in the following matrix form:

$$
\begin{aligned}
\left(\begin{array}{llll}
0 & 0 & 0 & 0 \\
0 & 1 & 0 & 0 \\
0 & 0 & 1 & 0 \\
0 & 0 & 0 & 1
\end{array}\right) \frac{\partial}{\partial t}\left(\begin{array}{c}
p \\
v \\
w \\
\theta
\end{array}\right)= & \left(\begin{array}{c}
\boldsymbol{\nabla} \cdot \boldsymbol{v} \\
-\boldsymbol{v} \cdot \nabla v-\partial_{x} p \\
-\boldsymbol{v} \cdot \nabla w-\partial_{y} p+\operatorname{Pr} \operatorname{Ra} \theta \\
-\boldsymbol{v} \cdot \nabla \theta+w
\end{array}\right) \\
& +\left(\begin{array}{cccc}
0 & 0 & 0 & 0 \\
0 & \operatorname{Pr} & 0 & 0 \\
0 & 0 & \operatorname{Pr} & 0 \\
0 & 0 & 0 & 1
\end{array}\right) \nabla^{2}\left(\begin{array}{c}
p \\
v \\
w \\
\theta
\end{array}\right) .
\end{aligned}
$$

Introducing the variable $\boldsymbol{X}(x, y, t)$ as

$$
\boldsymbol{X}(x, y, t)=(p, v, w, \theta)^{\mathrm{T}},
$$

Eq. (14) can be rewritten as

$$
\hat{M} \frac{\partial}{\partial t} \boldsymbol{X}(x, y, t)=\mathcal{F}[\boldsymbol{X}]+\hat{D} \nabla^{2} \boldsymbol{X}
$$

where the first term on the right-hand side is given by

$$
\mathcal{F}[X]=\left\{\begin{array}{l}
\boldsymbol{\nabla} \cdot \boldsymbol{v} \\
-\boldsymbol{v} \cdot \nabla v-\partial_{x} p \\
-\boldsymbol{v} \cdot \nabla w-\partial_{y} p+\operatorname{Pr} \operatorname{Ra} \theta \\
-\boldsymbol{v} \cdot \nabla \theta+w
\end{array}\right.
$$

and the two matrices are given by

$$
\hat{M}=\left(\begin{array}{llll}
0 & 0 & 0 & 0 \\
0 & 1 & 0 & 0 \\
0 & 0 & 1 & 0 \\
0 & 0 & 0 & 1
\end{array}\right), \quad \hat{D}=\left(\begin{array}{cccc}
0 & 0 & 0 & 0 \\
0 & \operatorname{Pr} & 0 & 0 \\
0 & 0 & \operatorname{Pr} & 0 \\
0 & 0 & 0 & 1
\end{array}\right)
$$

It is evident that the above matrices are singular. In particular, as $\hat{M}$ is singular, Eq. (16) can be considered as a partial differential algebraic equation or a partial differential equation with constraints (see, e.g., Refs. $[47,48]$ ). 


\section{B. Limit-torus solutions and their Floquet-type systems}

In general, a stable limit-torus solution of Eq. (16), which represents traveling and oscillating thermal convection, can be described as (e.g., see Figs. 2-4 in Sec. III A)

$$
\boldsymbol{X}(x, y, t)=\boldsymbol{X}_{0}(x-\Phi, y, \Theta), \quad \dot{\Phi}(t)=c, \quad \dot{\Theta}(t)=\omega .
$$

Here, the spatial and temporal phases are denoted by $\Phi$ and $\Theta$, respectively. These phases indicate the position and oscillation of the thermal convection, respectively. Further, the traveling velocity $c$ and oscillation frequency $\omega$ are constant. The limit-torus solution $\boldsymbol{X}_{0}(x-\Phi, y, \Theta)$ satisfies both the $2 L$ periodicity with respect to $(x-\Phi)$ and the $2 \pi$ periodicity with respect to $\Theta$, i.e.,

$$
\begin{aligned}
& \boldsymbol{X}_{0}(x-\Phi+2 L, y, \Theta)=\boldsymbol{X}_{0}(x-\Phi, y, \Theta), \\
& \boldsymbol{X}_{0}(x-\Phi, y, \Theta+2 \pi)=\boldsymbol{X}_{0}(x-\Phi, y, \Theta) .
\end{aligned}
$$

Substituting Eq. (19) into Eq. (16), we find that $\boldsymbol{X}_{0}(x-$ $\Phi, y, \Theta)$ satisfies the following equation:

$$
\left[-c \hat{M} \frac{\partial}{\partial x}+\omega \hat{M} \frac{\partial}{\partial \Theta}\right] \boldsymbol{X}_{0}(x-\Phi, y, \Theta)=\mathcal{F}\left[\boldsymbol{X}_{0}\right]+\hat{D} \nabla^{2} \boldsymbol{X}_{0}
$$

The components of $\boldsymbol{X}_{0}(x-\Phi, y, \Theta)$ can be defined as

$$
\boldsymbol{X}_{0}(x-\Phi, y, \Theta)=\left(p_{0}, v_{0}, w_{0}, \theta_{0}\right)^{\mathrm{T}} .
$$

Further, the components of $\boldsymbol{u}(x-\Phi, y, \Theta, t)$ are defined as

$$
\boldsymbol{u}(x-\Phi, y, \Theta, t)=\left(p_{1}, v_{1}, w_{1}, \theta_{1}\right)^{\mathrm{T}} \text {. }
$$

Here, we consider that $\boldsymbol{u}(x-\Phi, y, \Theta, t)$ represents a small disturbance to $\boldsymbol{X}_{0}(x-\Phi, y, \Theta)$. Under this condition, the small perturbation solution can be expressed as

$$
\boldsymbol{X}(x, y, t)=\boldsymbol{X}_{0}(x-\Phi, y, \Theta)+\boldsymbol{u}(x-\Phi, y, \Theta, t) .
$$

Equation (16) is then linearized with respect to $\boldsymbol{u}(x-$ $\Phi, y, \Theta, t)$ as follows:

$$
\hat{M} \frac{\partial}{\partial t} \boldsymbol{u}(x-\Phi, y, \Theta, t)=\hat{\mathcal{L}}(x-\Phi, y, \Theta) \boldsymbol{u}(x-\Phi, y, \Theta, t) .
$$

Here, the linear operator $\hat{\mathcal{L}}(x-\Phi, y, \Theta)$ is given by

$$
\begin{aligned}
& \hat{\mathcal{L}}(x-\Phi, y, \Theta) \boldsymbol{u}(x-\Phi, y, \Theta) \\
& \quad=\left[\hat{L}(x-\Phi, y, \Theta)+c \hat{M} \frac{\partial}{\partial x}-\omega \hat{M} \frac{\partial}{\partial \Theta}\right] \boldsymbol{u}(x-\Phi, y, \Theta),
\end{aligned}
$$

where

$$
\begin{aligned}
& \hat{L}(x-\Phi, y, \Theta) \boldsymbol{u}(x-\Phi, y, \Theta) \\
& \quad=\left[\hat{\mathcal{J}}(x-\Phi, y, \Theta)+\hat{D} \nabla^{2}\right] \boldsymbol{u}(x-\Phi, y, \Theta) .
\end{aligned}
$$

The explicit form of $\hat{\mathcal{J}}(x-\Phi, y, \Theta) \boldsymbol{u}(x-\Phi, y, \Theta)$, which can be derived from $\mathcal{F}[\boldsymbol{X}]$, is given by Eq. (A7) in Appendix A. In Eqs. (27) and (28), we omitted the $t$ dependence of the function $\boldsymbol{u}(x-\Phi, y, \Theta, t)$ and denoted it as $\boldsymbol{u}(x-\Phi, y, \Theta)$ because we consider only the eigenvalue problem of the linear operator $\hat{\mathcal{L}}(x-\Phi, y, \Theta)$. As in the limit-torus solution $\boldsymbol{X}_{0}(x-\Phi, y, \Theta)$, the function $\boldsymbol{u}(x-\Phi, y, \Theta)$ satisfies the $2 L$ periodicity with respect to $(x-\Phi)$, the Dirichlet zero boundary condition with respect to $y$, and the $2 \pi$ periodicity with respect to $\Theta$. Note that the linear operator $\hat{\mathcal{L}}(x-\Phi, y, \Theta)$ is periodic with respect to both $(x-\Phi)$ and $\Theta$. Therefore, Eq. (26) represents a Floquet-type system with two zero eigenvalues, which are associated with spatial and temporal translational symmetry breaking.

As in Eq. (24), we define the components of $\boldsymbol{u}^{*}(x-$ $\Phi, y, \Theta)$ as

$$
\boldsymbol{u}^{*}(x-\Phi, y, \Theta)=\left(p_{1}^{*}, v_{1}^{*}, w_{1}^{*}, \theta_{1}^{*}\right)
$$

Further, we also define the inner product of two functions as

$$
\begin{aligned}
& \llbracket \boldsymbol{u}^{*}(x-\Phi, y, \Theta), \boldsymbol{u}(x-\Phi, y, \Theta) \rrbracket \\
& \quad=\frac{1}{2 \pi} \int_{0}^{2 \pi} d \Theta \int_{0}^{2 L} d x \int_{-1}^{+1} d y \boldsymbol{u}^{*}(x-\Phi, y, \Theta) \cdot \boldsymbol{u}(x-\Phi, y, \Theta),
\end{aligned}
$$

where $\quad \boldsymbol{u}^{*}(x-\Phi, y, \Theta) \cdot \boldsymbol{u}(x-\Phi, y, \Theta)=p_{1}^{*} p_{1}+v_{1}^{*} v_{1}+$ $w_{1}^{*} w_{1}+\theta_{1}^{*} \theta_{1}$, using Eqs. (24) and (29). We introduce the adjoint operator of $\hat{\mathcal{L}}(x-\Phi, y, \Theta)$ as

$$
\begin{aligned}
& \llbracket \boldsymbol{u}^{*}(x-\Phi, y, \Theta), \hat{\mathcal{L}}(x-\Phi, y, \Theta) \boldsymbol{u}(x-\Phi, y, \Theta) \rrbracket \\
& =\llbracket \hat{\mathcal{L}}^{*}(x-\Phi, y, \Theta) \boldsymbol{u}^{*}(x-\Phi, y, \Theta), \boldsymbol{u}(x-\Phi, y, \Theta) \rrbracket \\
& \quad+\mathcal{S}\left[\boldsymbol{u}^{*}(x-\Phi, y, \Theta), \boldsymbol{u}(x-\Phi, y, \Theta)\right],
\end{aligned}
$$

where the bilinear concomitant is denoted by $\mathcal{S}\left[\boldsymbol{u}^{*}(x-\right.$ $\Phi, y, \Theta), \boldsymbol{u}(x-\Phi, y, \Theta)]$. Using partial integration, the adjoint operator $\hat{\mathcal{L}}^{*}(x-\Phi, y, \Theta)$ can be expressed as

$$
\begin{aligned}
& \hat{\mathcal{L}}^{*}(x-\Phi, y, \Theta) \boldsymbol{u}^{*}(x-\Phi, y, \Theta) \\
& \quad=\left[\hat{L}^{*}(x-\Phi, y, \Theta)-c \hat{M}^{\mathrm{T}} \frac{\partial}{\partial x}+\omega \hat{M}^{\mathrm{T}} \frac{\partial}{\partial \Theta}\right] \boldsymbol{u}^{*}(x-\Phi, y, \Theta),
\end{aligned}
$$

where

$$
\begin{aligned}
& \hat{L}^{*}(x-\Phi, y, \Theta) \boldsymbol{u}^{*}(x-\Phi, y, \Theta) \\
& \quad=\left[\hat{\mathcal{J}}^{*}(x-\Phi, y, \Theta)+\hat{D}^{\mathrm{T}} \nabla^{2}\right] \boldsymbol{u}^{*}(x-\Phi, y, \Theta) .
\end{aligned}
$$

The explicit form of $\hat{\mathcal{J}}^{*}(x-\Phi, y, \Theta) \boldsymbol{u}^{*}(x-\Phi, y, \Theta)$, which can be derived from $\hat{\mathcal{J}}(x-\Phi, y, \Theta) \boldsymbol{u}(x-\Phi, y, \Theta)$, is given by Eq. (A10) in Appendix A. As in $\boldsymbol{u}(x-\Phi, y, \Theta)$, the function $\boldsymbol{u}^{*}(x-\Phi, y, \Theta)$ also satisfies the $2 L$ periodicity with respect to $(x-\Phi)$, the Dirichlet zero boundary condition with respect to $y$, and the $2 \pi$ periodicity with respect to $\Theta$. Details of the adjoint operator including the adjoint boundary conditions are given in Appendix A.

\section{Floquet zero eigenfunctions and their orthonormalization}

Here, we use the Floquet and adjoint eigenfunctions associated with the two zero eigenvalues of $\hat{\mathcal{L}}(x-\Phi, y, \Theta)$ and $\hat{\mathcal{L}}^{*}(x-\Phi, y, \Theta)$. From Eqs. (27) and (32), the zero 
eigenfunctions satisfy the following conditions:

$$
\begin{aligned}
\hat{\mathcal{L}}(x-\Phi, y, \Theta) \boldsymbol{U}_{\mathrm{s}}(x-\Phi, y, \Theta) & =\left[\hat{L}(x-\Phi, y, \Theta)+c \hat{M} \frac{\partial}{\partial x}-\omega \hat{M} \frac{\partial}{\partial \Theta}\right] \boldsymbol{U}_{\mathrm{s}}(x-\Phi, y, \Theta)=0, \\
\hat{\mathcal{L}}(x-\Phi, y, \Theta) \boldsymbol{U}_{\mathrm{t}}(x-\Phi, y, \Theta) & =\left[\hat{L}(x-\Phi, y, \Theta)+c \hat{M} \frac{\partial}{\partial x}-\omega \hat{M} \frac{\partial}{\partial \Theta}\right] \boldsymbol{U}_{\mathrm{t}}(x-\Phi, y, \Theta)=0, \\
\hat{\mathcal{L}}^{*}(x-\Phi, y, \Theta) \boldsymbol{U}_{\mathrm{s}}^{*}(x-\Phi, y, \Theta) & =\left[\hat{L}^{*}(x-\Phi, y, \Theta)-c \hat{M}^{\mathrm{T}} \frac{\partial}{\partial x}+\omega \hat{M}^{\mathrm{T}} \frac{\partial}{\partial \Theta}\right] \boldsymbol{U}_{\mathrm{s}}^{*}(x-\Phi, y, \Theta)=0, \\
\hat{\mathcal{L}}^{*}(x-\Phi, y, \Theta) \boldsymbol{U}_{\mathrm{t}}^{*}(x-\Phi, y, \Theta) & =\left[\hat{L}^{*}(x-\Phi, y, \Theta)-c \hat{M}^{\mathrm{T}} \frac{\partial}{\partial x}+\omega \hat{M}^{\mathrm{T}} \frac{\partial}{\partial \Theta}\right] \boldsymbol{U}_{\mathrm{t}}^{*}(x-\Phi, y, \Theta)=0 .
\end{aligned}
$$

Note that the two Floquet zero eigenfunctions, i.e., $\boldsymbol{U}_{\mathrm{s}}(x-\Phi, y, \Theta)$ for the spatial phase and $\boldsymbol{U}_{\mathrm{t}}(x-\Phi, y, \Theta)$ for the temporal phase, can be chosen as (e.g., see Figs. 6-11 in Sec. III B)

$$
\begin{aligned}
\boldsymbol{U}_{\mathrm{s}}(x-\Phi, y, \Theta) & =\frac{\partial}{\partial x} \boldsymbol{X}_{0}(x-\Phi, y, \Theta), \\
\boldsymbol{U}_{\mathrm{t}}(x-\Phi, y, \Theta) & =\frac{\partial}{\partial \Theta} \boldsymbol{X}_{0}(x-\Phi, y, \Theta),
\end{aligned}
$$

which can be confirmed by differentiating Eq. (22) with respect to $x$ and $\Theta$, respectively. For the inner product given by Eq. (30) with the two Floquet zero eigenfunctions given by Eqs. (38) and (39), the corresponding two adjoint zero eigenfunctions, i.e., $\boldsymbol{U}_{\mathrm{s}}^{*}(x-\Phi, y, \Theta)$ for the spatial phase and $\boldsymbol{U}_{\mathrm{t}}^{*}(x-\Phi, y, \Theta)$ for the temporal phase, can be orthonormalized as

$$
\llbracket \boldsymbol{U}_{\mathrm{p}}^{*}(x-\Phi, y, \Theta), \hat{M} \boldsymbol{U}_{\mathrm{q}}(x-\Phi, y, \Theta) \rrbracket=\frac{1}{2 \pi} \int_{0}^{2 \pi} d \Theta \int_{0}^{2 L} d x \int_{-1}^{+1} d y \boldsymbol{U}_{\mathrm{p}}^{*}(x-\Phi, y, \Theta) \cdot \hat{M} \boldsymbol{U}_{\mathrm{q}}(x-\Phi, y, \Theta)=\delta_{\mathrm{pq}},
$$

where $\mathrm{p}, \mathrm{q}=\mathrm{s}$, t. Note that the singular diagonal matrix $\hat{M}=\operatorname{diag}(0,1,1,1)$, which is defined in Eq. (18), has been inserted in Eq. (40) because of the form of Eq. (16). This is the key point of the proposed phase reduction method for partial differential algebraic equations. Here, we also note that the following condition is satisfied:

$$
\begin{aligned}
& \frac{\partial}{\partial \Theta}\left[\int_{0}^{2 L} d x \int_{-1}^{+1} d y \boldsymbol{U}_{\mathrm{p}}^{*}(x-\Phi, y, \Theta) \cdot \hat{M} \boldsymbol{U}_{\mathrm{q}}(x-\Phi, y, \Theta)\right] \\
& =\int_{0}^{2 L} d x \int_{-1}^{+1} d y\left[\boldsymbol{U}_{\mathrm{p}}^{*}(x-\Phi, y, \Theta) \cdot \hat{M} \frac{\partial}{\partial \Theta} \boldsymbol{U}_{\mathrm{q}}(x-\Phi, y, \Theta)+\hat{M}^{\mathrm{T}} \frac{\partial}{\partial \Theta} \boldsymbol{U}_{\mathrm{p}}^{*}(x-\Phi, y, \Theta) \cdot \boldsymbol{U}_{\mathrm{q}}(x-\Phi, y, \Theta)\right] \\
& =\frac{1}{\omega} \int_{0}^{2 L} d x \int_{-1}^{+1} d y\left[\boldsymbol{U}_{\mathrm{p}}^{*}(x-\Phi, y, \Theta) \cdot\left\{\hat{L}(x-\Phi, y, \Theta)+c \hat{M} \frac{\partial}{\partial x}\right\} \boldsymbol{U}_{\mathrm{q}}(x-\Phi, y, \Theta)\right. \\
& \left.\quad-\left\{\hat{L}^{*}(x-\Phi, y, \Theta)-c \hat{M}^{\mathrm{T}} \frac{\partial}{\partial x}\right\} \boldsymbol{U}_{\mathrm{p}}^{*}(x-\Phi, y, \Theta) \cdot \boldsymbol{U}_{\mathrm{q}}(x-\Phi, y, \Theta)\right] \\
& =0,
\end{aligned}
$$

where we used Eqs. (34), (35), (36), and (37). This implies that the following orthonormalization condition is satisfied independently for each $\Theta$ :

$$
\int_{0}^{2 L} d x \int_{-1}^{+1} d y \boldsymbol{U}_{\mathrm{p}}^{*}(x-\Phi, y, \Theta) \cdot \hat{M} \boldsymbol{U}_{\mathrm{q}}(x-\Phi, y, \Theta)=\delta_{\mathrm{pq}} .
$$

Here, we describe a numerical method for obtaining the adjoint zero eigenfunctions (see Appendix $\mathrm{C}$ for more details). From Eqs. (36) and (37), the adjoint zero eigenfunctions satisfy

$$
\omega \hat{M}^{\mathrm{T}} \frac{\partial}{\partial \Theta} \boldsymbol{U}_{\mathrm{p}}^{*}(x-\Phi, y, \Theta)=-\left[\hat{\mathcal{J}}^{*}(x-\Phi, y, \Theta)+\hat{D}^{\mathrm{T}} \nabla^{2}-c \hat{M}^{\mathrm{T}} \frac{\partial}{\partial x}\right] \boldsymbol{U}_{\mathrm{p}}^{*}(x-\Phi, y, \Theta),
$$

for $\mathrm{p}=\mathrm{s}, \mathrm{t}$, which can be transformed as

$$
\hat{M}^{\mathrm{T}} \frac{\partial}{\partial s} \boldsymbol{U}_{\mathrm{p}}^{*}(x-\Phi, y,-\omega s)=\left[\hat{\mathcal{J}}^{*}(x-\Phi, y,-\omega s)+\hat{D}^{\mathrm{T}} \nabla^{2}-c \hat{M}^{\mathrm{T}} \frac{\partial}{\partial x}\right] \boldsymbol{U}_{\mathrm{p}}^{*}(x-\Phi, y,-\omega s)
$$

by substituting $\Theta=-\omega s$. A relaxation method using Eq. (44), which can also be called the adjoint method (see Refs. [6-12] for limit-cycle solutions to ordinary differential equations, Refs. [20-26] for limit-cycle solutions to partial differential equations, and Ref. [46] for limit-torus solutions to partial differential equations), is convenient to obtain the adjoint zero eigenfunctions associated with limit-torus solutions to partial differential algebraic equations. 
In the following two subsections, we derive a set of phase equations for traveling and oscillating thermal convection using the limit-torus solution, its Floquet zero eigenfunctions, and adjoint zero eigenfunctions.

\section{Traveling and oscillating convection with weak perturbations}

Here, we consider traveling and oscillating thermal convection with a weak perturbation that is described by the following equation:

$$
\hat{M} \frac{\partial}{\partial t} \boldsymbol{X}(x, y, t)=\mathcal{F}[\boldsymbol{X}]+\hat{D} \nabla^{2} \boldsymbol{X}+\epsilon \boldsymbol{P}(x, y, t) .
$$

The weak perturbation is denoted by $\epsilon \boldsymbol{P}(x, y, t)$. We assume that the perturbed solution is always near the orbit of the limit-torus solution $\boldsymbol{X}_{0}(x-\Phi, y, \Theta)$. Using the two adjoint zero eigenfunctions, we project the dynamics of the perturbed equation (45) onto the unperturbed limit-torus solution with respect to the spatial and temporal phases as follows:

$$
\begin{aligned}
-\dot{\Phi}(t) & =\int_{0}^{2 L} d x \int_{-1}^{+1} d y \boldsymbol{U}_{\mathrm{s}}^{*}(x-\Phi, y, \Theta) \cdot\left[\hat{M} \frac{\partial}{\partial t} \boldsymbol{X}(x, y, t)\right] \\
& =\int_{0}^{2 L} d x \int_{-1}^{+1} d y \boldsymbol{U}_{\mathrm{s}}^{*}(x-\Phi, y, \Theta) \cdot\left[\mathcal{F}[\boldsymbol{X}]+\hat{D} \nabla^{2} \boldsymbol{X}+\epsilon \boldsymbol{P}(x, y, t)\right] \\
& \simeq \int_{0}^{2 L} d x \int_{-1}^{+1} d y \boldsymbol{U}_{\mathrm{s}}^{*}(x-\Phi, y, \Theta) \cdot\left[\mathcal{F}\left[\boldsymbol{X}_{0}\right]+\hat{D} \nabla^{2} \boldsymbol{X}_{0}+\epsilon \boldsymbol{P}(x, y, t)\right] \\
& =\int_{0}^{2 L} d x \int_{-1}^{+1} d y \boldsymbol{U}_{\mathrm{s}}^{*}(x-\Phi, y, \Theta) \cdot\left[-c \hat{M} \frac{\partial \boldsymbol{X}_{0}}{\partial x}+\omega \hat{M} \frac{\partial \boldsymbol{X}_{0}}{\partial \Theta}+\epsilon \boldsymbol{P}(x, y, t)\right] \\
& =\int_{0}^{2 L} d x \int_{-1}^{+1} d y \boldsymbol{U}_{\mathrm{s}}^{*}(x-\Phi, y, \Theta) \cdot\left[-c \hat{M} \boldsymbol{U}_{\mathrm{s}}(x-\Phi, y, \Theta)+\omega \hat{M} \boldsymbol{U}_{\mathrm{t}}(x-\Phi, y, \Theta)+\epsilon \boldsymbol{P}(x, y, t)\right] \\
& =-c+\epsilon \int_{0}^{2 L} d x \int_{-1}^{+1} d y \boldsymbol{U}_{\mathrm{s}}^{*}(x-\Phi, y, \Theta) \cdot \boldsymbol{P}(x, y, t),
\end{aligned}
$$

and

$$
\begin{aligned}
\dot{\Theta}(t) & =\int_{0}^{2 L} d x \int_{-1}^{+1} d y \boldsymbol{U}_{\mathrm{t}}^{*}(x-\Phi, y, \Theta) \cdot\left[\hat{M} \frac{\partial}{\partial t} \boldsymbol{X}(x, y, t)\right] \\
& =\int_{0}^{2 L} d x \int_{-1}^{+1} d y \boldsymbol{U}_{\mathrm{t}}^{*}(x-\Phi, y, \Theta) \cdot\left[\mathcal{F}[\boldsymbol{X}]+\hat{D} \nabla^{2} \boldsymbol{X}+\epsilon \boldsymbol{P}(x, y, t)\right] \\
& \simeq \int_{0}^{2 L} d x \int_{-1}^{+1} d y \boldsymbol{U}_{\mathrm{t}}^{*}(x-\Phi, y, \Theta) \cdot\left[\mathcal{F}\left[\boldsymbol{X}_{0}\right]+\hat{D} \nabla^{2} \boldsymbol{X}_{0}+\epsilon \boldsymbol{P}(x, y, t)\right] \\
& =\int_{0}^{2 L} d x \int_{-1}^{+1} d y \boldsymbol{U}_{\mathrm{t}}^{*}(x-\Phi, y, \Theta) \cdot\left[-c \hat{M} \frac{\partial \boldsymbol{X}_{0}}{\partial x}+\omega \hat{M} \frac{\partial \boldsymbol{X}_{0}}{\partial \Theta}+\epsilon \boldsymbol{P}(x, y, t)\right] \\
& =\int_{0}^{2 L} d x \int_{-1}^{+1} d y \boldsymbol{U}_{\mathrm{t}}^{*}(x-\Phi, y, \Theta) \cdot\left[-c \hat{M} \boldsymbol{U}_{\mathrm{s}}(x-\Phi, y, \Theta)+\omega \hat{M} \boldsymbol{U}_{\mathrm{t}}(x-\Phi, y, \Theta)+\epsilon \boldsymbol{P}(x, y, t)\right] \\
& =\omega+\epsilon \int_{0}^{2 L} d x \int_{-1}^{+1} d y \boldsymbol{U}_{\mathrm{t}}^{*}(x-\Phi, y, \Theta) \cdot \boldsymbol{P}(x, y, t),
\end{aligned}
$$

where we approximated $\boldsymbol{X}(x, y, t)$ by the unperturbed limit-torus solution $\boldsymbol{X}_{0}(x-\Phi, y, \Theta)$, and also used Eqs. (22), (38), (39), and (42). Consequently, the two phase equations describing the traveling and oscillating thermal convection under weak perturbation are approximately obtained in the following forms:

$$
\begin{aligned}
& \dot{\Phi}(t)=c+\epsilon \int_{0}^{2 L} d x \int_{-1}^{+1} d y \boldsymbol{Z}_{\mathrm{s}}(x-\Phi, y, \Theta) \cdot \boldsymbol{P}(x, y, t), \\
& \dot{\Theta}(t)=\omega+\epsilon \int_{0}^{2 L} d x \int_{-1}^{+1} d y \boldsymbol{Z}_{\mathrm{t}}(x-\Phi, y, \Theta) \cdot \boldsymbol{P}(x, y, t),
\end{aligned}
$$

where the phase sensitivity functions for the spatial and temporal phases are defined as (e.g., see Figs. 12-17 in Sec. III B)

$$
\begin{aligned}
& Z_{\mathrm{s}}(x-\Phi, y, \Theta)=-\boldsymbol{U}_{\mathrm{s}}^{*}(x-\Phi, y, \Theta), \\
& \boldsymbol{Z}_{\mathrm{t}}(x-\Phi, y, \Theta)=+\boldsymbol{U}_{\mathrm{t}}^{*}(x-\Phi, y, \Theta) .
\end{aligned}
$$


These phase equations are the main results of this paper. The phase sensitivity functions quantify the spatiotemporal phase responses of the thermal convection to weak perturbations applied at each spatial point and at each time. The coupling of spatial and temporal phases, as clear from Eqs. (48) and (49), manifests in a nontrivial spatiotemporal phase dynamics.

Here, the components of the phase sensitivity functions for the spatial and temporal phases are defined as

$$
\begin{gathered}
Z_{\mathrm{s}}(x-\Phi, y, \Theta)=\left(Z_{p}^{(\mathrm{s})}, Z_{v}^{(\mathrm{s})}, Z_{w}^{(\mathrm{s})}, Z_{\theta}^{(\mathrm{s})}\right), \\
Z_{\mathrm{t}}(x-\Phi, y, \Theta)=\left(Z_{p}^{(\mathrm{t})}, Z_{v}^{(\mathrm{t})}, Z_{w}^{(\mathrm{t})}, Z_{\theta}^{(\mathrm{t})}\right) .
\end{gathered}
$$

As is clear from Eq. (A18) in Appendix A, not only the fluid velocity field $\boldsymbol{v}$ but also its phase sensitivity functions are divergence-free, which can be expressed as follows:

$$
\begin{gathered}
\partial_{x} Z_{v}^{(\mathrm{s})}+\partial_{y} Z_{w}^{(\mathrm{s})}=0, \\
\partial_{x} Z_{v}^{(\mathrm{t})}+\partial_{y} Z_{w}^{(\mathrm{t})}=0 .
\end{gathered}
$$

Here, we consider scalar-potential-based perturbations to the fluid velocity field as follows:

$$
\boldsymbol{P}(x, y, t)=\left(0,-\partial_{x} \Psi,-\partial_{y} \Psi, 0\right)^{\mathrm{T}},
$$

where the scalar potential is denoted by $\Psi$. In this case, the spatial and temporal phase responses given in Eqs. (48) and (49) become zero, i.e.,

$$
\begin{gathered}
\int_{0}^{2 L} d x \int_{-1}^{+1} d y \boldsymbol{Z}_{\mathrm{s}}(x-\Phi, y, \Theta) \cdot \boldsymbol{P}(x, y, t) \\
=-\int_{0}^{2 L} d x \int_{-1}^{+1} d y\left(Z_{v}^{(\mathrm{s})} \partial_{x} \Psi+Z_{w}^{(\mathrm{s})} \partial_{y} \Psi\right) \\
=\int_{0}^{2 L} d x \int_{-1}^{+1} d y\left(\partial_{x} Z_{v}^{(\mathrm{s})}+\partial_{y} Z_{w}^{(\mathrm{s})}\right) \Psi=0, \\
\int_{0}^{2 L} d x \int_{-1}^{+1} d y Z_{\mathrm{t}}(x-\Phi, y, \Theta) \cdot \boldsymbol{P}(x, y, t) \\
=-\int_{0}^{2 L} d x \int_{-1}^{+1} d y\left(Z_{v}^{(\mathrm{t})} \partial_{x} \Psi+Z_{w}^{(\mathrm{t})} \partial_{y} \Psi\right) \\
=\int_{0}^{2 L} d x \int_{-1}^{+1} d y\left(\partial_{x} Z_{v}^{(\mathrm{t})}+\partial_{y} Z_{w}^{(\mathrm{t})}\right) \Psi=0,
\end{gathered}
$$

where we used Eqs. (54), (55), and (A15). This implies that application of scalar-potential-based perturbations to the fluid velocity field does not affect the spatial and temporal phases. Here, we note that such a perturbation in incompressible fluid equations can be absorbed into the pressure term, which is clear from the following equations:

$$
\begin{gathered}
0=\nabla \cdot \boldsymbol{v}, \\
\frac{\partial}{\partial t} \boldsymbol{v}(x, y, t)=-\boldsymbol{v} \cdot \nabla \boldsymbol{v}-\nabla p+\operatorname{Pr} \operatorname{Ra} \theta \boldsymbol{e}_{y}+\operatorname{Pr} \nabla^{2} \boldsymbol{v}-\epsilon \nabla \Psi,
\end{gathered}
$$$$
\frac{\partial}{\partial t} \theta(x, y, t)=-\boldsymbol{v} \cdot \nabla \theta+\boldsymbol{v} \cdot \boldsymbol{e}_{y}+\nabla^{2} \theta,
$$

where $\boldsymbol{e}_{y}$ is a unit vector for $y$. Therefore, the divergence-free property described by Eqs. (54) and (55) holds naturally and universally for incompressible fluids.
We now consider the case when the perturbation can be described by a product of two functions, i.e.,

$$
\boldsymbol{P}(x, y, t)=\boldsymbol{A}(x, y) q(t) .
$$

This signifies that the space dependence and time dependence of the perturbation are separated. In this case, Eqs. (48) and (49) can be written as

$$
\begin{aligned}
& \dot{\Phi}(t)=c+\epsilon \zeta_{\mathrm{s}}(\Phi, \Theta) q(t), \\
& \dot{\Theta}(t)=\omega+\epsilon \zeta_{\mathrm{t}}(\Phi, \Theta) q(t),
\end{aligned}
$$

where the effective phase sensitivity functions for the spatial and temporal phases are expressed as

$$
\begin{aligned}
& \zeta_{\mathrm{s}}(\Phi, \Theta)=\int_{0}^{2 L} d x \int_{-1}^{+1} d y \boldsymbol{Z}_{\mathrm{s}}(x-\Phi, y, \Theta) \cdot \boldsymbol{A}(x, y), \\
& \zeta_{\mathrm{t}}(\Phi, \Theta)=\int_{0}^{2 L} d x \int_{-1}^{+1} d y \boldsymbol{Z}_{\mathrm{t}}(x-\Phi, y, \Theta) \cdot \boldsymbol{A}(x, y) .
\end{aligned}
$$

We note that the forms of Eqs. (63) and (64) are essentially the same as those of the phase equations, which were derived for a perturbed limit-torus oscillator described by a finitedimensional dynamical system (see Ref. [49]). This implies that a traveling and oscillating solution to a partial differential algebraic equation can be reduced to a set of phase equations, as in an ordinary limit-torus oscillator.

\section{E. Weakly coupled systems of traveling and oscillating convection}

Here, we consider weakly coupled systems of traveling and oscillating thermal convection that are described by the following equation:

$$
\hat{M} \frac{\partial}{\partial t} \boldsymbol{X}_{\sigma}(x, y, t)=\mathcal{F}\left[\boldsymbol{X}_{\sigma}\right]+\hat{D} \nabla^{2} \boldsymbol{X}_{\sigma}+\epsilon \mathcal{G}\left[\boldsymbol{X}_{\sigma}, \boldsymbol{X}_{\tau}\right],
$$

for $(\sigma, \tau)=(1,2)$ or $(2,1)$, where the weak coupling term is denoted by $\epsilon \mathcal{G}\left[\boldsymbol{X}_{\sigma}, \boldsymbol{X}_{\tau}\right]$. In the absence of coupling, each system is assumed to exhibit a stable limit-torus solution given as

$$
\boldsymbol{X}_{\sigma}(x, y, t)=\boldsymbol{X}_{0}\left(x-\Phi_{\sigma}, y, \Theta_{\sigma}\right), \quad \dot{\Phi}_{\sigma}(t)=c, \quad \dot{\Theta}_{\sigma}(t)=\omega .
$$

Here, we assume that the coupling between the systems is sufficiently weak. Under these assumptions, as in Sec. II D, we can obtain a set of phase equations from Eq. (67) as follows:

$$
\begin{aligned}
\dot{\Phi}_{\sigma}(t)= & c+\epsilon \int_{0}^{2 L} d x \int_{-1}^{+1} d y \boldsymbol{Z}_{\mathrm{s}}\left(x-\Phi_{\sigma}, y, \Theta_{\sigma}\right) \\
& \cdot \mathcal{G}\left[\boldsymbol{X}_{0}\left(x-\Phi_{\sigma}, y, \Theta_{\sigma}\right), \boldsymbol{X}_{0}\left(x-\Phi_{\tau}, y, \Theta_{\tau}\right)\right] \\
\equiv & c+\epsilon \widetilde{\Gamma}_{\mathrm{s}}\left(\Phi_{\sigma}-\Phi_{\tau}, \Theta_{\sigma}, \Theta_{\tau}\right), \\
\dot{\Theta}_{\sigma}(t)= & \omega+\epsilon \int_{0}^{2 L} d x \int_{-1}^{+1} d y \boldsymbol{Z}_{\mathrm{t}}\left(x-\Phi_{\sigma}, y, \Theta_{\sigma}\right) \\
& \cdot \mathcal{G}\left[\boldsymbol{X}_{0}\left(x-\Phi_{\sigma}, y, \Theta_{\sigma}\right), \boldsymbol{X}_{0}\left(x-\Phi_{\tau}, y, \Theta_{\tau}\right)\right] \\
\equiv & \omega+\epsilon \widetilde{\Gamma}_{\mathrm{t}}\left(\Phi_{\sigma}-\Phi_{\tau}, \Theta_{\sigma}, \Theta_{\tau}\right),
\end{aligned}
$$

where we approximated $\boldsymbol{X}_{\sigma}(x, y, t)$ by the unperturbed limittorus solution $\boldsymbol{X}_{0}\left(x-\Phi_{\sigma}, y, \Theta_{\sigma}\right)$. These two functions, i.e., 
$\tilde{\Gamma}_{\mathrm{s}}\left(\Phi_{\sigma}-\Phi_{\tau}, \Theta_{\sigma}, \Theta_{\tau}\right)$ for the spatial phase and $\tilde{\Gamma}_{\mathrm{t}}\left(\Phi_{\sigma}-\right.$ $\Phi_{\tau}, \Theta_{\sigma}, \Theta_{\tau}$ ) for the temporal phase, depend on the spatial phase difference between the systems and the temporal phases of both systems.

Introducing the slow phase variables as

$$
\begin{aligned}
& \Phi_{\sigma}(t)=c t+\bar{\Phi}_{\sigma}(t), \\
& \Theta_{\sigma}(t)=\omega t+\bar{\Theta}_{\sigma}(t),
\end{aligned}
$$

we can rewrite Eqs. (69) and (70) as

$$
\begin{aligned}
& \frac{d}{d t} \bar{\Phi}_{\sigma}(t)=\epsilon \widetilde{\Gamma}_{\mathrm{s}}\left(\bar{\Phi}_{\sigma}-\bar{\Phi}_{\tau}, \omega t+\bar{\Theta}_{\sigma}, \omega t+\bar{\Theta}_{\tau}\right), \\
& \frac{d}{d t} \bar{\Theta}_{\sigma}(t)=\epsilon \widetilde{\Gamma}_{\mathrm{t}}\left(\bar{\Phi}_{\sigma}-\bar{\Phi}_{\tau}, \omega t+\bar{\Theta}_{\sigma}, \omega t+\bar{\Theta}_{\tau}\right) .
\end{aligned}
$$

When we apply the averaging method [2] with respect to the temporal phases, Eqs. (73) and (74) can be written as follows:

$$
\begin{aligned}
\frac{d}{d t} \bar{\Phi}_{\sigma}(t) & =\frac{\epsilon}{2 \pi} \int_{0}^{2 \pi} d \lambda \widetilde{\Gamma}_{\mathrm{s}}\left(\bar{\Phi}_{\sigma}-\bar{\Phi}_{\tau}, \lambda+\bar{\Theta}_{\sigma}, \lambda+\bar{\Theta}_{\tau}\right) \\
& \equiv \epsilon \Gamma_{\mathrm{s}}\left(\bar{\Phi}_{\sigma}-\bar{\Phi}_{\tau}, \bar{\Theta}_{\sigma}-\bar{\Theta}_{\tau}\right), \\
\frac{d}{d t} \bar{\Theta}_{\sigma}(t) & =\frac{\epsilon}{2 \pi} \int_{0}^{2 \pi} d \lambda \widetilde{\Gamma}_{\mathrm{t}}\left(\bar{\Phi}_{\sigma}-\bar{\Phi}_{\tau}, \lambda+\bar{\Theta}_{\sigma}, \lambda+\bar{\Theta}_{\tau}\right) \\
& \equiv \epsilon \Gamma_{\mathrm{t}}\left(\bar{\Phi}_{\sigma}-\bar{\Phi}_{\tau}, \bar{\Theta}_{\sigma}-\bar{\Theta}_{\tau}\right) .
\end{aligned}
$$

Therefore, we obtain the following phase equations:

$$
\begin{aligned}
& \dot{\Phi}_{\sigma}(t)=c+\epsilon \Gamma_{\mathrm{s}}\left(\Phi_{\sigma}-\Phi_{\tau}, \Theta_{\sigma}-\Theta_{\tau}\right), \\
& \dot{\Theta}_{\sigma}(t)=\omega+\epsilon \Gamma_{\mathrm{t}}\left(\Phi_{\sigma}-\Phi_{\tau}, \Theta_{\sigma}-\Theta_{\tau}\right),
\end{aligned}
$$

where the phase coupling functions for the spatial and temporal phases can be described as

$$
\begin{aligned}
\Gamma_{\mathrm{s}}(\Phi, \Theta)= & \frac{1}{2 \pi} \int_{0}^{2 \pi} d \lambda \int_{0}^{2 L} d x \int_{-1}^{+1} d y \boldsymbol{Z}_{\mathrm{s}}(x-\Phi, y, \lambda+\Theta) \\
& \cdot \mathcal{G}\left[\boldsymbol{X}_{0}(x-\Phi, y, \lambda+\Theta), \boldsymbol{X}_{0}(x, y, \lambda)\right], \\
\Gamma_{\mathrm{t}}(\Phi, \Theta)= & \frac{1}{2 \pi} \int_{0}^{2 \pi} d \lambda \int_{0}^{2 L} d x \int_{-1}^{+1} d y \boldsymbol{Z}_{\mathrm{t}}(x-\Phi, y, \lambda+\Theta) \\
& \cdot \mathcal{G}\left[\boldsymbol{X}_{0}(x-\Phi, y, \lambda+\Theta), \boldsymbol{X}_{0}(x, y, \lambda)\right] .
\end{aligned}
$$

The phase coupling functions, i.e., $\Gamma_{\mathrm{s}}\left(\Phi_{\sigma}-\Phi_{\tau}, \Theta_{\sigma}-\Theta_{\tau}\right)$ for the spatial phase and $\Gamma_{\mathrm{t}}\left(\Phi_{\sigma}-\Phi_{\tau}, \Theta_{\sigma}-\Theta_{\tau}\right)$ for the temporal phase, depend only on the spatial and temporal phase differences.

Let the spatial and temporal phase differences be defined as

$$
\begin{aligned}
& \Delta \Phi(t)=\Phi_{1}(t)-\Phi_{2}(t), \\
& \Delta \Theta(t)=\Theta_{1}(t)-\Theta_{2}(t) .
\end{aligned}
$$

Using Eqs. (77) and (78), we obtain the following equations,

$$
\begin{aligned}
& \frac{d}{d t} \Delta \Phi(t)=\epsilon \Gamma_{\mathrm{s}}^{(\mathrm{a})}(\Delta \Phi, \Delta \Theta), \\
& \frac{d}{d t} \Delta \Theta(t)=\epsilon \Gamma_{\mathrm{t}}^{(\mathrm{a})}(\Delta \Phi, \Delta \Theta),
\end{aligned}
$$

where the antisymmetric components of the phase coupling functions are defined as (e.g., see Figs. 18 and 19 in Sec. III C)

$$
\begin{aligned}
& \Gamma_{\mathrm{s}}^{(\mathrm{a})}(\Delta \Phi, \Delta \Theta)=\Gamma_{\mathrm{s}}(\Delta \Phi, \Delta \Theta)-\Gamma_{\mathrm{s}}(-\Delta \Phi,-\Delta \Theta), \\
& \Gamma_{\mathrm{t}}^{(\mathrm{a})}(\Delta \Phi, \Delta \Theta)=\Gamma_{\mathrm{t}}(\Delta \Phi, \Delta \Theta)-\Gamma_{\mathrm{t}}(-\Delta \Phi,-\Delta \Theta) .
\end{aligned}
$$

These two functions, $\Gamma_{\mathrm{s}}^{(\mathrm{a})}(\Delta \Phi, \Delta \Theta)$ and $\Gamma_{\mathrm{t}}^{(\mathrm{a})}(\Delta \Phi, \Delta \Theta)$, satisfy the following properties:

$$
\begin{aligned}
& \Gamma_{\mathrm{s}}^{(\mathrm{a})}(-\Delta \Phi,-\Delta \Theta)=-\Gamma_{\mathrm{s}}^{(\mathrm{a})}(\Delta \Phi, \Delta \Theta), \\
& \Gamma_{\mathrm{t}}^{(\mathrm{a})}(-\Delta \Phi,-\Delta \Theta)=-\Gamma_{\mathrm{t}}^{(\mathrm{a})}(\Delta \Phi, \Delta \Theta),
\end{aligned}
$$

which represent the antisymmetry with respect to the origin, i.e., $\Delta \Phi=\Delta \Theta=0$.

Finally, we note that the forms of Eqs. (77) and (78) are the same as that of the phase equations that are derived for weakly coupled limit-torus oscillators described by finite-dimensional dynamical systems (see Ref. [49]). This implies that, as in an ordinary limit-torus oscillator, weakly coupled systems with traveling and oscillating solutions to a partial differential algebraic equation can be reduced to a set of phase equations.

\section{NUMERICAL ANALYSIS OF LIMIT-TORUS SOLUTIONS}

In this section, we perform a numerical analysis of traveling and oscillating thermal convection to illustrate the theory formulated in Sec. II. The numerical methods for fluid equations and adjoint equations are summarized in Appendixes B and $\mathrm{C}$, respectively. The number of grid points for $x, y$, and $\Theta$ are $N_{x}=256, N_{y}+1=129$, and $N_{\Theta}=1024$, respectively. The time step is $\Delta t=10^{-4}-10^{-5}$ (primarily, $\Delta t=10^{-4}$ ). The parameters are fixed, i.e., $\operatorname{Pr}=6.8, \mathrm{Ra}=5000$, and $2 L=4$. The value of $\operatorname{Pr}$ is the typical one for water at room temperature. The values of the others were chosen such that the system can exhibit oscillatory thermal convection. The initial values were prepared such that the system exhibits oscillatory thermal convection with a pair of vortices. The traveling velocity and oscillation frequency are $c=0$ and $\omega \simeq 108$, respectively. As $c=0$, the traveling and oscillating thermal convection can be considered as oscillatory thermal convection with a spatially translational mode. ${ }^{1}$

\section{A. Thermal convection solutions}

The components of the limit-torus solution $\boldsymbol{X}_{0}(x-$ $\Phi, y, \Theta)$ are defined as

$$
\boldsymbol{X}_{0}(x-\Phi, y, \Theta)=\left(p_{0}, v_{0}, w_{0}, \theta_{0}\right)^{\mathrm{T}}, \quad \boldsymbol{v}_{0}=\left(v_{0}, w_{0}\right)^{\mathrm{T}},
$$

\footnotetext{
${ }^{1}$ We have not found a case of nonzero traveling velocity in our numerical simulations of this system although we have varied the Prandtl number, Rayleigh number, and aspect ratio (i.e., Pr, Ra, and $L$ ). Therefore, we consider only the case of zero traveling velocity in this paper. Here, we note that the theory formulated in Sec. II is applicable for the case of non-zero traveling velocity. This point is further discussed in Sec. IV.
} 

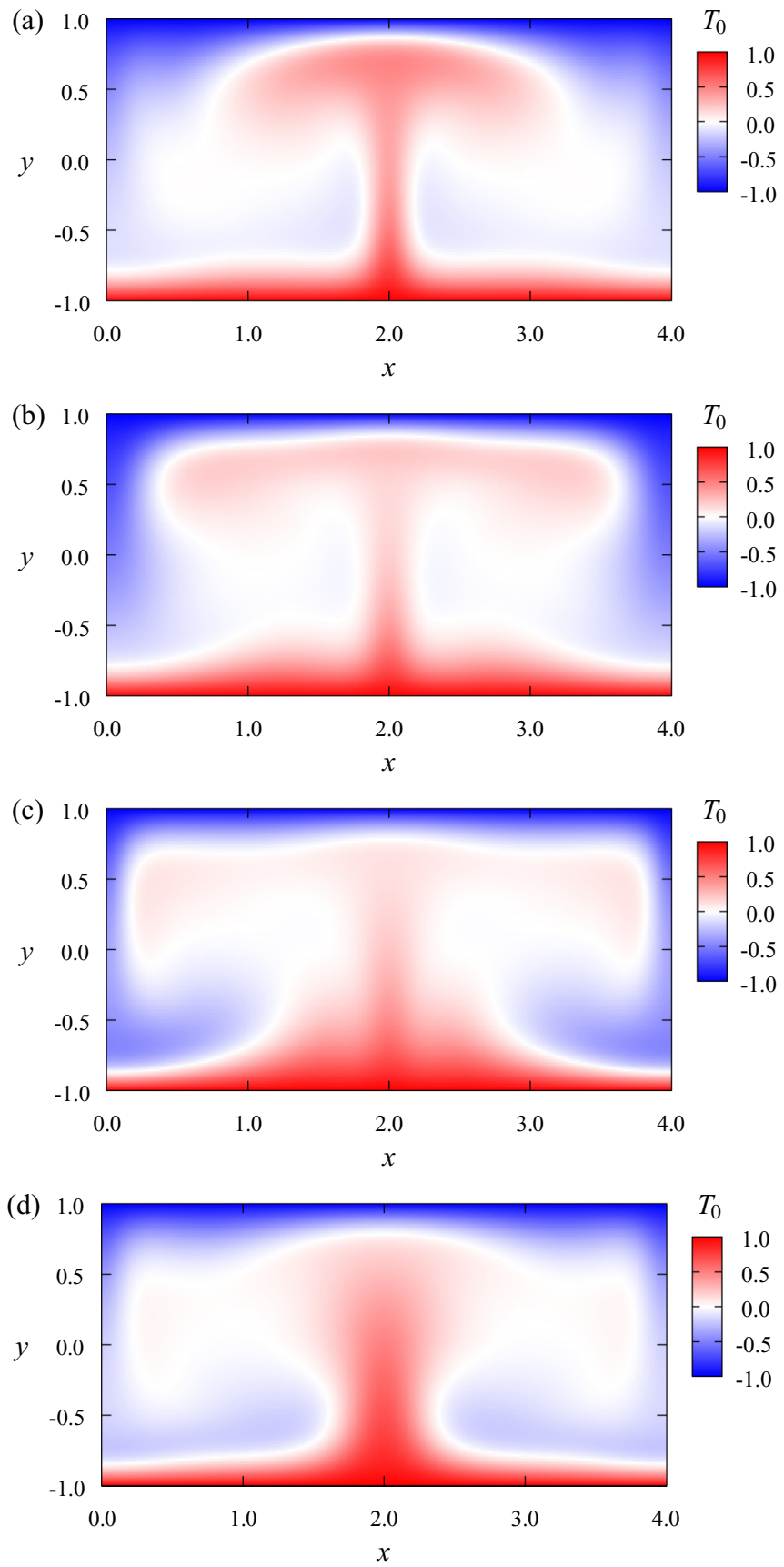

FIG. 1. Snapshots of $T_{0}(x-\Phi, y, \Theta)$ with $\Phi=2$ for (a) $\Theta / \pi=$ 0.0 , (b) $\Theta / \pi=0.5$, (c) $\Theta / \pi=1.0$, and (d) $\Theta / \pi=1.5$.

where the convective component of the temperature field is defined as

$$
T_{0}(x-\Phi, y, \Theta)=-y+\theta_{0}(x-\Phi, y, \Theta) .
$$

Figure 1 shows the snapshots of the temperature field $T_{0}(x-$ $\Phi, y, \Theta)$ with $\Phi=2$ for [Fig. 1(a)] $\Theta / \pi=0.0$, [Fig. 1(b)] $\Theta / \pi=0.5$, [Fig. 1(c)] $\Theta / \pi=1.0$, and [Fig. $1(\mathrm{~d})] \Theta / \pi=$ 1.5. Figure 2 shows the snapshots of the convective component $\theta_{0}(x-\Phi, y, \Theta)$. Figures 3 and 4 show the snapshots of the horizontal fluid velocity $v_{0}(x-\Phi, y, \Theta)$ and the vertical fluid velocity $w_{0}(x-\Phi, y, \Theta)$, respectively. Equation (1) suggests that the fluid velocity field is incompressible or
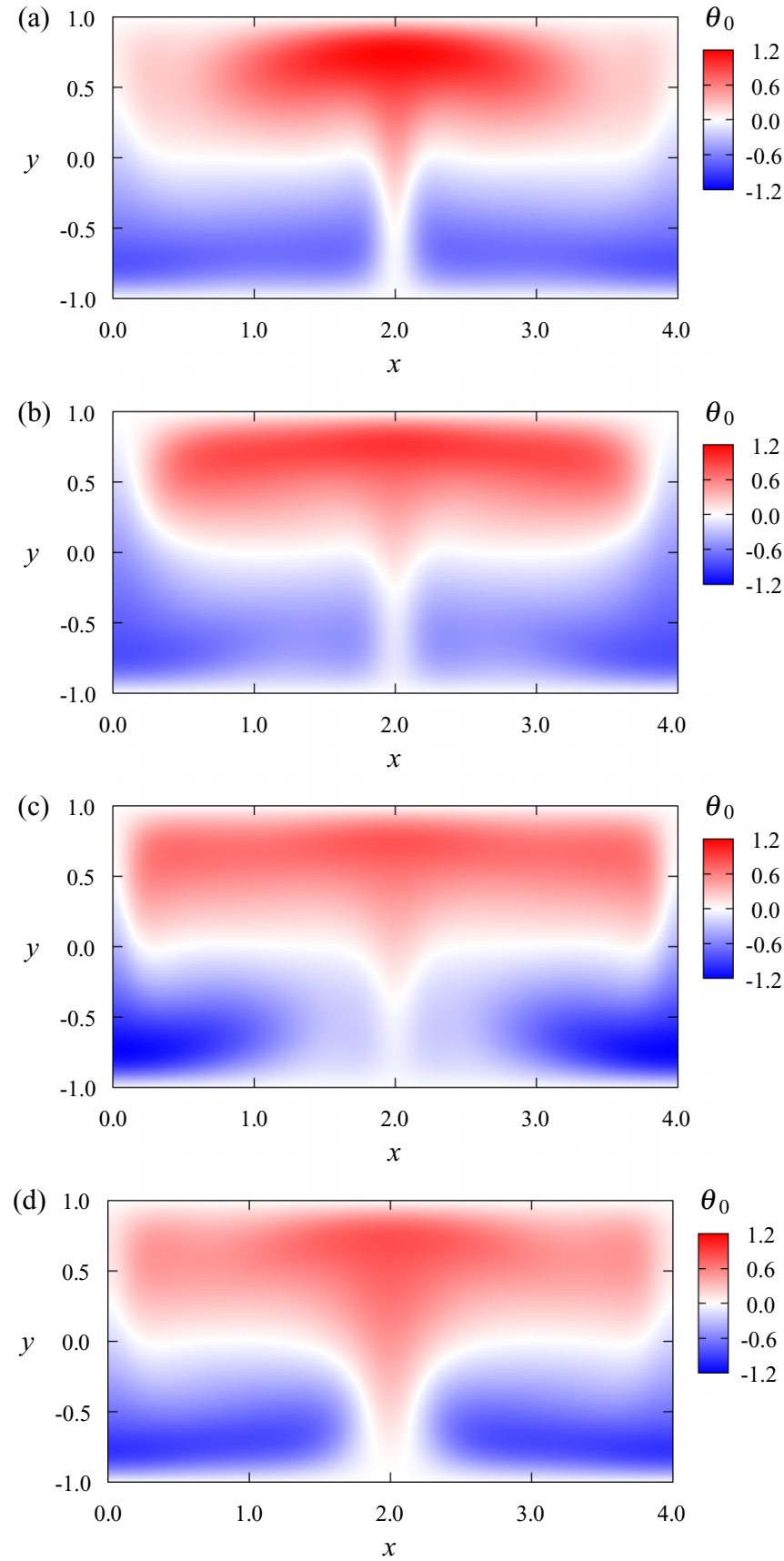

FIG. 2. Snapshots of $\theta_{0}(x-\Phi, y, \Theta)$ with $\Phi=2$ for (a) $\Theta / \pi=$ 0.0 , (b) $\Theta / \pi=0.5$, (c) $\Theta / \pi=1.0$, and (d) $\Theta / \pi=1.5$.

divergence-free (see Figs. 3 and 4), i.e.,

$$
\partial_{x} v_{0}+\partial_{y} w_{0}=0 \text {. }
$$

Because the traveling velocity is zero, i.e.,

$$
c=0 \text {, }
$$

the limit-torus solution $\boldsymbol{X}_{0}(x-\Phi, y, \Theta)$, representing the oscillatory thermal convection, exhibits the following reflection properties (see Figs. 2-4):

$$
\begin{aligned}
\theta_{0}(-(x-\Phi), y, \Theta) & =+\theta_{0}(x-\Phi, y, \Theta), \\
v_{0}(-(x-\Phi), y, \Theta) & =-v_{0}(x-\Phi, y, \Theta), \\
w_{0}(-(x-\Phi), y, \Theta) & =+w_{0}(x-\Phi, y, \Theta) .
\end{aligned}
$$



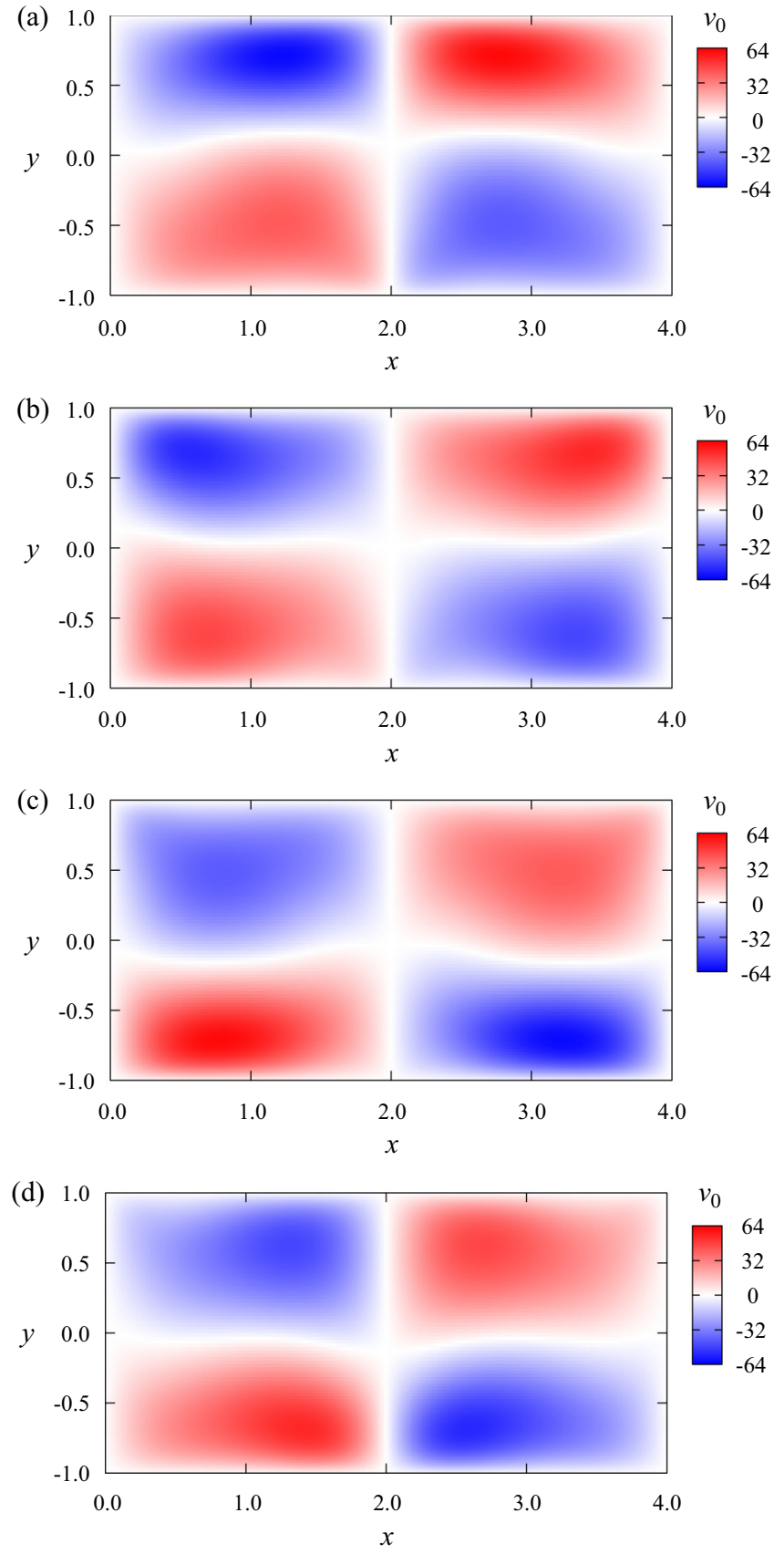

FIG. 3. Snapshots of $v_{0}(x-\Phi, y, \Theta)$ with $\Phi=2$ for (a) $\Theta / \pi=$ 0.0 , (b) $\Theta / \pi=0.5$, (c) $\Theta / \pi=1.0$, and (d) $\Theta / \pi=1.5$.

For visualizing the temporal phase of the limit-torus in the infinite-dimensional state space, we project the limit-torus solution $\boldsymbol{X}_{0}(x-\Phi, y, \Theta)$ onto the following quantity with $k=3$ :

$$
H_{k}(\Theta)=\int_{0}^{2 L} d x \int_{-1}^{+1} d y \theta_{0}(x-\Phi, y, \Theta) \sin \left(k \pi \frac{y+1}{2}\right),
$$

which is a real number and depends only on the temporal phase $\Theta$. Figure 5 shows $H_{3}(\Theta)$, which satisfies the following property: $H_{3}(\Theta+\pi)=-H_{3}(\Theta)$. To determine the spatial phase of the limit-torus solution $\boldsymbol{X}_{0}(x-\Phi, y, \Theta)$, we
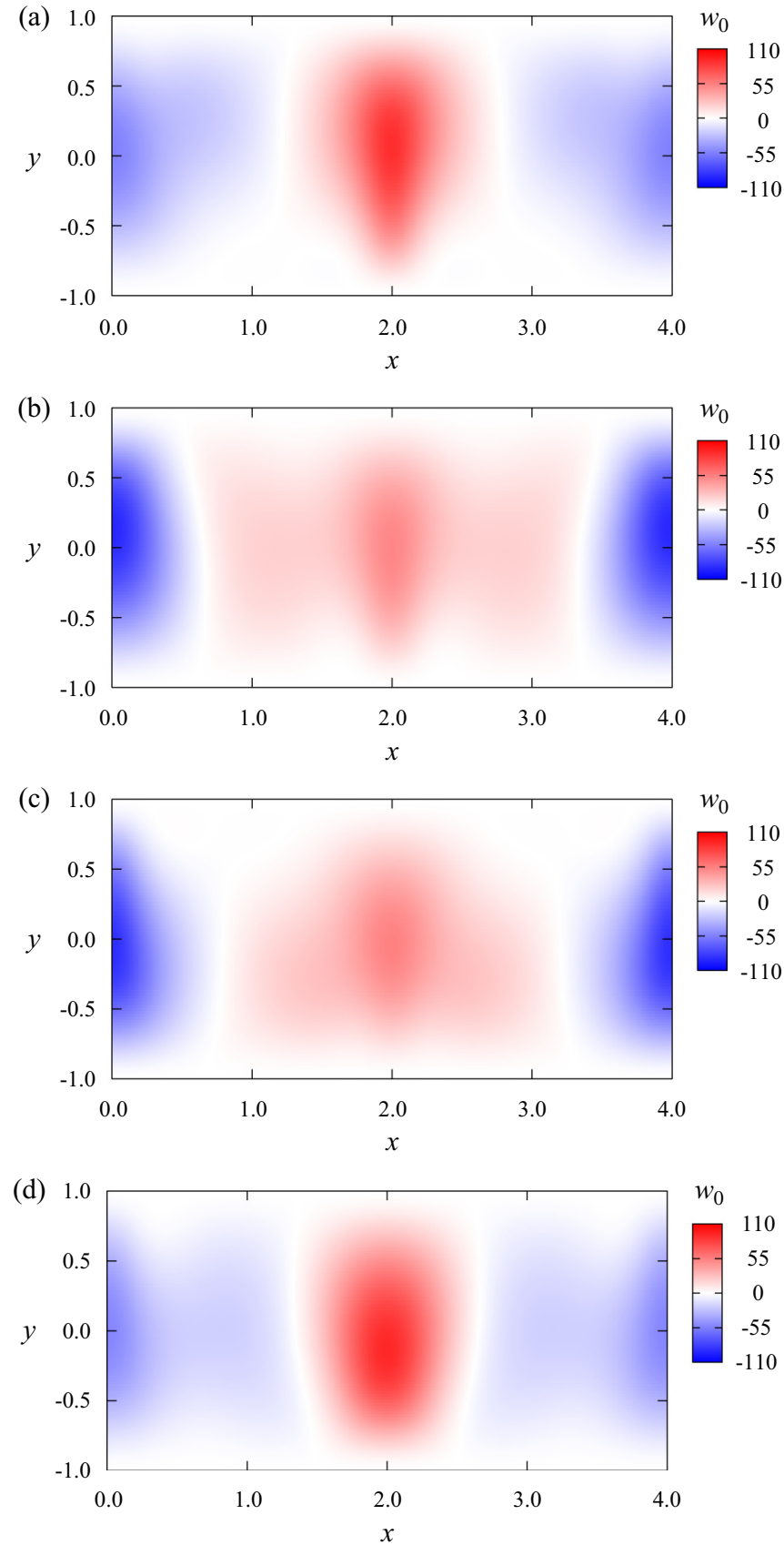

FIG. 4. Snapshots of $w_{0}(x-\Phi, y, \Theta)$ with $\Phi=2$ for (a) $\Theta / \pi=$ 0.0, (b) $\Theta / \pi=0.5$, (c) $\Theta / \pi=1.0$, and (d) $\Theta / \pi=1.5$.

introduce the following complex parameter:

$$
\begin{aligned}
A(\Phi, \Theta)= & \int_{0}^{2 L} d x \int_{-1}^{+1} d y \theta_{0}(x-\Phi, y, \Theta) \\
& \times \exp \left(i \pi \frac{x}{L}\right) \sin \left(\pi \frac{y+1}{2}\right) .
\end{aligned}
$$

Consequently, the spatial phase $\Phi$ is given as

$$
\frac{\Phi}{L}=\frac{\arg A}{\pi} \text {. }
$$

As seen in Fig. 1, the spatial phase $\Phi$ indicates the position of the hot plume in the thermal convection, whereas the temporal phase $\Theta$ indicates its oscillation. 


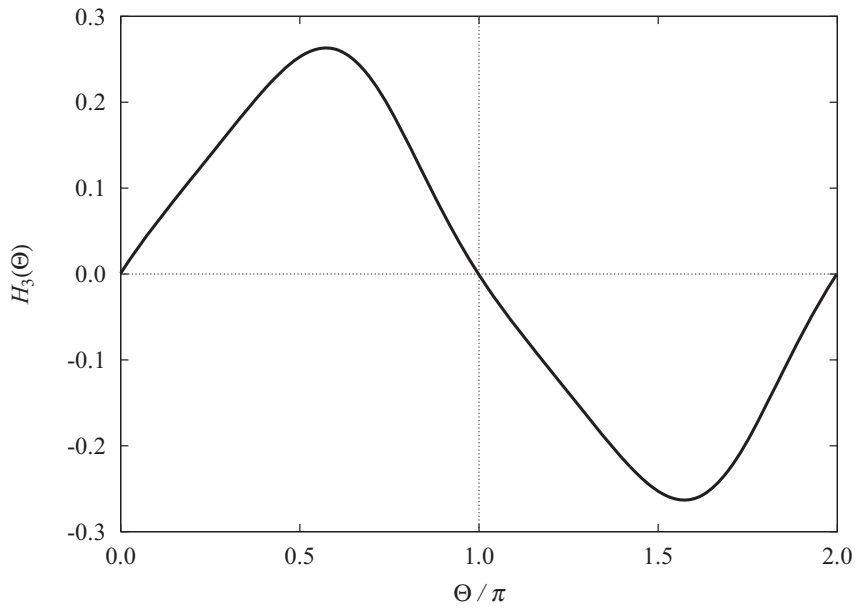

FIG. 5. Wave form of $H_{3}(\Theta)$. The parameters are fixed, i.e., $\operatorname{Pr}=$ $6.8, \mathrm{Ra}=5000$, and $2 L=4$. The traveling velocity and oscillation frequency are $c=0$ and $\omega \simeq 108$, respectively.

\section{B. Phase sensitivity functions}

The components of the Floquet zero eigenfunctions for the spatial and temporal phases are defined as

$$
\begin{aligned}
& U_{\mathrm{s}}(x-\Phi, y, \Theta)=\left(U_{p}^{(\mathrm{s})}, U_{v}^{(\mathrm{s})}, U_{w}^{(\mathrm{s})}, U_{\theta}^{(\mathrm{s})}\right)^{\mathrm{T}}, \\
& \boldsymbol{U}_{\mathrm{t}}(x-\Phi, y, \Theta)=\left(U_{p}^{(\mathrm{t})}, U_{v}^{(\mathrm{t})}, U_{w}^{(\mathrm{t})}, U_{\theta}^{(\mathrm{t})}\right)^{\mathrm{T}} .
\end{aligned}
$$

Figures 6, 7, and 8 show the snapshots of the Floquet zero eigenfunction for the spatial phase, $U_{\theta}^{(\mathrm{s})}(x-\Phi, y, \Theta)$, $U_{v}^{(\mathrm{s})}(x-\Phi, y, \Theta)$, and $U_{w}^{(\mathrm{s})}(x-\Phi, y, \Theta)$, respectively. Figures 9, 10, and 11 show the snapshots of the Floquet zero eigenfunction for the temporal phase, $U_{\theta}^{(\mathrm{t})}(x-\Phi, y, \Theta)$, $U_{v}^{(\mathrm{t})}(x-\Phi, y, \Theta)$, and $U_{w}^{(\mathrm{t})}(x-\Phi, y, \Theta)$, respectively.

As in Eqs. (52) and (53), the components of the phase sensitivity functions for the spatial and temporal phases are defined as

$$
\begin{aligned}
& Z_{\mathrm{s}}(x-\Phi, y, \Theta)=\left(Z_{p}^{(\mathrm{s})}, Z_{v}^{(\mathrm{s})}, Z_{w}^{(\mathrm{s})}, Z_{\theta}^{(\mathrm{s})}\right), \\
& Z_{\mathrm{t}}(x-\Phi, y, \Theta)=\left(Z_{p}^{(\mathrm{t})}, Z_{v}^{(\mathrm{t})}, Z_{w}^{(\mathrm{t})}, Z_{\theta}^{(\mathrm{t})}\right) .
\end{aligned}
$$

Figures 12, 13, and 14 show the snapshots of the phase sensitivity function for the spatial phase, $Z_{\theta}^{(\mathrm{s})}(x-\Phi, y, \Theta)$, $Z_{v}^{(\mathrm{s})}(x-\Phi, y, \Theta)$, and $Z_{w}^{(\mathrm{s})}(x-\Phi, y, \Theta)$, respectively. Figures 15,16 , and 17 show the snapshots of the phase sensitivity function for the temporal phase, $Z_{\theta}^{(\mathrm{t})}(x-\Phi, y, \Theta), Z_{v}^{(\mathrm{t})}(x-$ $\Phi, y, \Theta)$, and $Z_{w}^{(\mathrm{t})}(x-\Phi, y, \Theta)$, respectively.

From Eqs. (50), (51), and (42), the following orthonormalization conditions can be obtained (see Figs. 6-17):

$$
\begin{aligned}
& -\int_{0}^{2 L} d x \int_{-1}^{+1} d y\left[Z_{v}^{(\mathrm{s})} U_{v}^{(\mathrm{s})}+Z_{w}^{(\mathrm{s})} U_{w}^{(\mathrm{s})}+Z_{\theta}^{(\mathrm{s})} U_{\theta}^{(\mathrm{s})}\right]=1 \\
& -\int_{0}^{2 L} d x \int_{-1}^{+1} d y\left[Z_{v}^{(\mathrm{s})} U_{v}^{(\mathrm{t})}+Z_{w}^{(\mathrm{s})} U_{w}^{(\mathrm{t})}+Z_{\theta}^{(\mathrm{s})} U_{\theta}^{(\mathrm{t})}\right]=0
\end{aligned}
$$
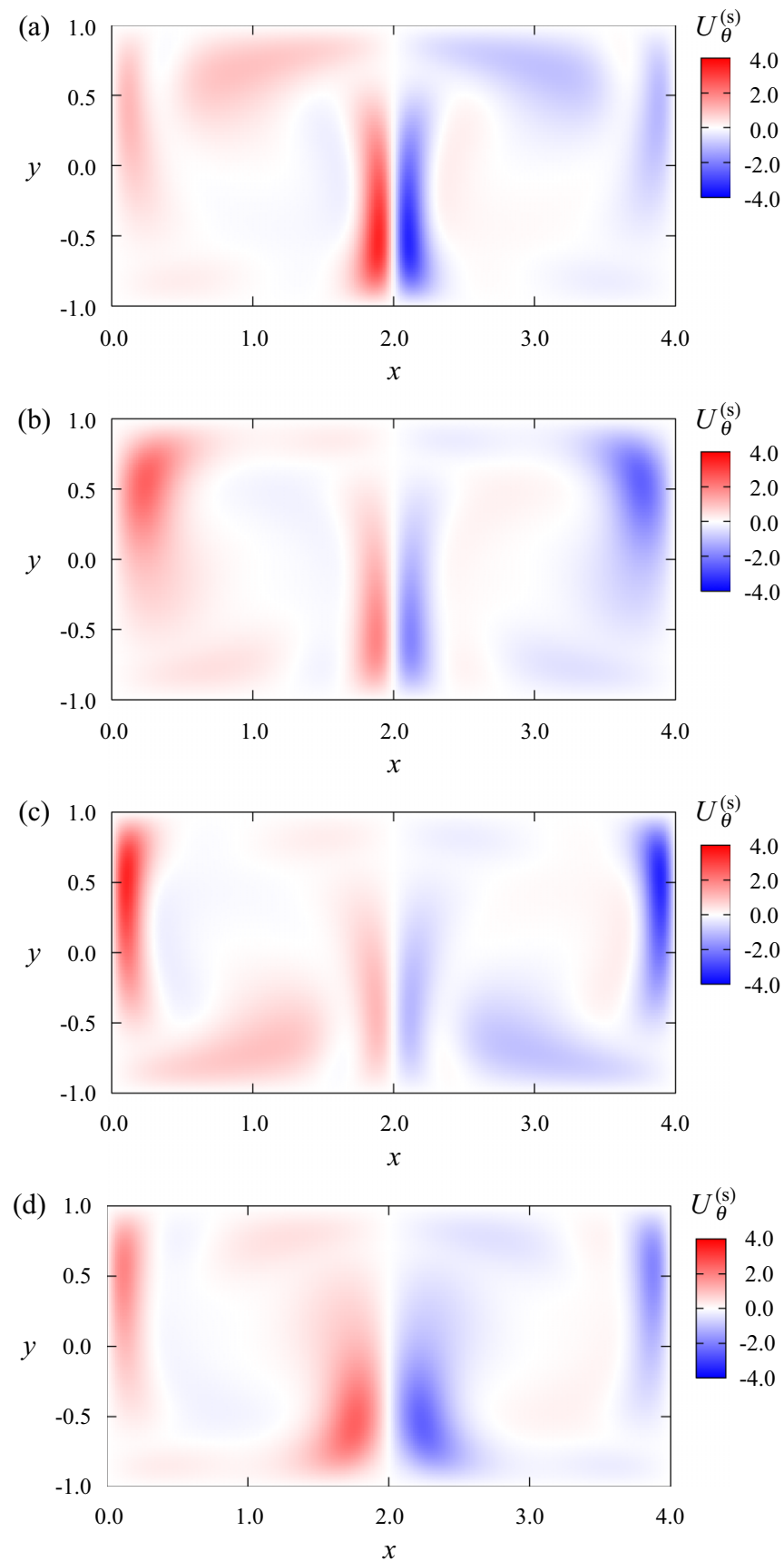

FIG. 6. Snapshots of $U_{\theta}^{(\mathrm{s})}(x-\Phi, y, \Theta)$ with $\Phi=2$ for (a) $\Theta / \pi=0.0$, (b) $\Theta / \pi=0.5$, (c) $\Theta / \pi=1.0$, and (d) $\Theta / \pi=1.5$.

$$
\begin{aligned}
& +\int_{0}^{2 L} d x \int_{-1}^{+1} d y\left[Z_{v}^{(\mathrm{t})} U_{v}^{(\mathrm{s})}+Z_{w}^{(\mathrm{t})} U_{w}^{(\mathrm{s})}+Z_{\theta}^{(\mathrm{t})} U_{\theta}^{(\mathrm{s})}\right]=0 \\
& +\int_{0}^{2 L} d x \int_{-1}^{+1} d y\left[Z_{v}^{(\mathrm{t})} U_{v}^{(\mathrm{t})}+Z_{w}^{(\mathrm{t})} U_{w}^{(\mathrm{t})}+Z_{\theta}^{(\mathrm{t})} U_{\theta}^{(\mathrm{t})}\right]=1
\end{aligned}
$$

where we used Eqs. (99), (100), (101), (102), and (18). From Eqs. (38), (39), and (91), the Floquet zero eigenfunctions 

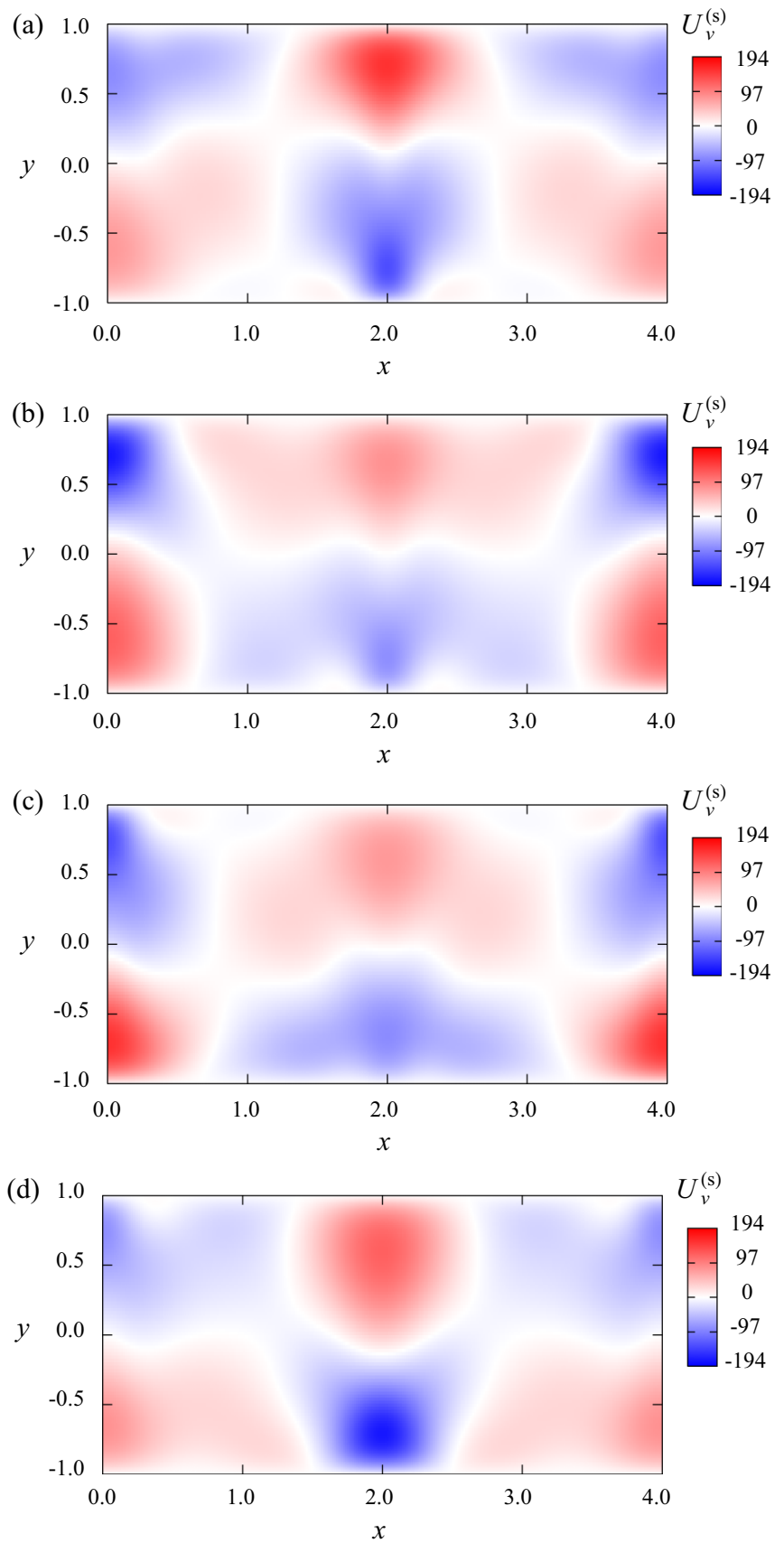

FIG. 7. Snapshots of $U_{v}^{(\mathrm{s})}(x-\Phi, y, \Theta)$ with $\Phi=2$ for (a) $\Theta / \pi=0.0$, (b) $\Theta / \pi=0.5$, (c) $\Theta / \pi=1.0$, and (d) $\Theta / \pi=1.5$.

for the spatial and temporal phases are divergence-free (see Figs. 7, 8, 10, and 11), i.e.,

$$
\begin{aligned}
& \partial_{x} U_{v}^{(\mathrm{s})}+\partial_{y} U_{w}^{(\mathrm{s})}=0, \\
& \partial_{x} U_{v}^{(\mathrm{t})}+\partial_{y} U_{w}^{(\mathrm{t})}=0 .
\end{aligned}
$$

As mentioned also in context of Eqs. (54) and (55), the phase sensitivity functions for the spatial and temporal phases are also divergence-free (see Figs. 13, 14, 16, and
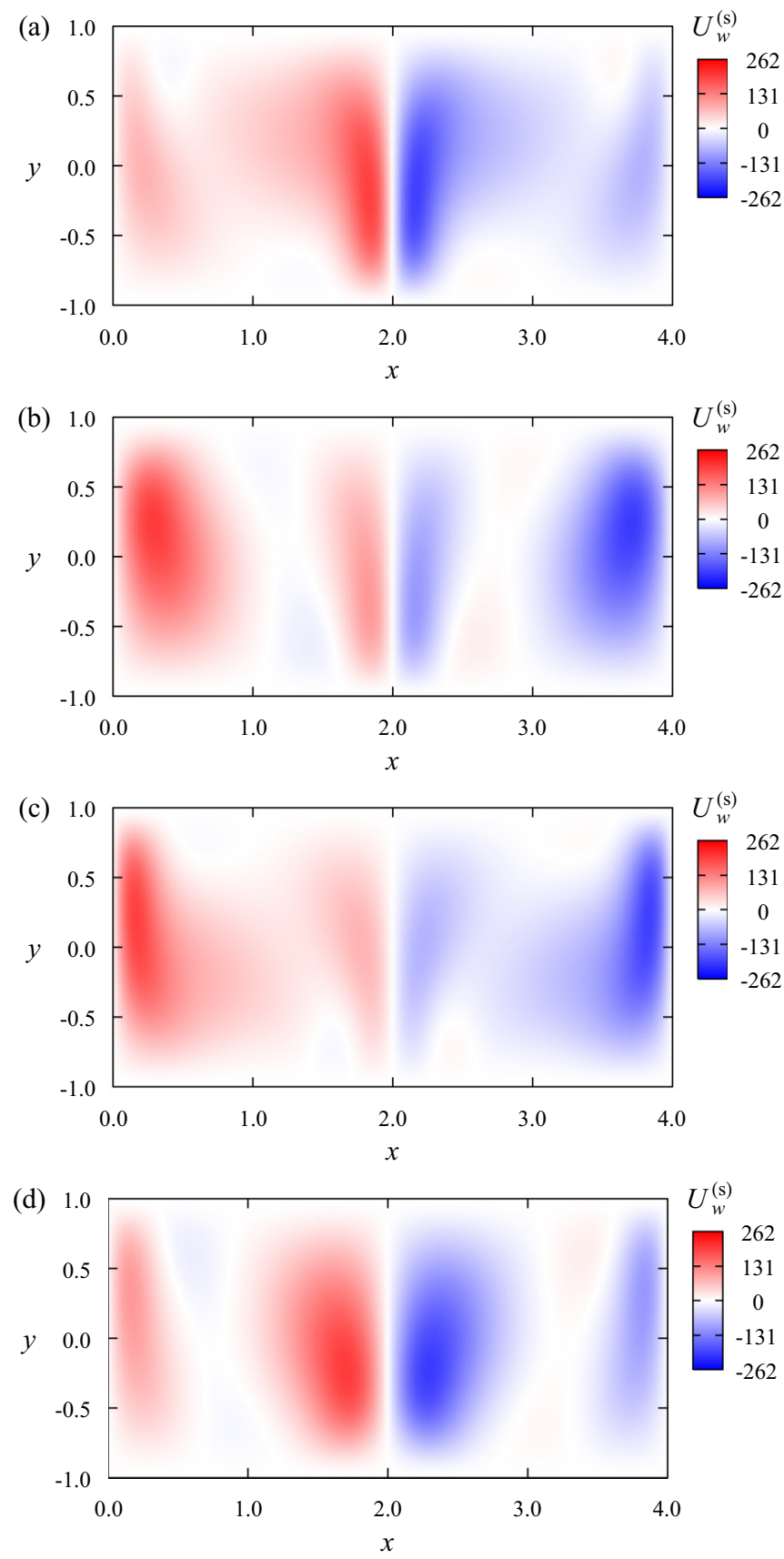

FIG. 8. Snapshots of $U_{w}^{(\mathrm{s})}(x-\Phi, y, \Theta)$ with $\Phi=2$ for (a) $\Theta / \pi=0.0$, (b) $\Theta / \pi=0.5$, (c) $\Theta / \pi=1.0$, and (d) $\Theta / \pi=1.5$.

17), i.e.,

$$
\begin{gathered}
\partial_{x} Z_{v}^{(\mathrm{s})}+\partial_{y} Z_{w}^{(\mathrm{s})}=0, \\
\partial_{x} Z_{v}^{(\mathrm{t})}+\partial_{y} Z_{w}^{(\mathrm{t})}=0 .
\end{gathered}
$$

From Eqs. (93), (94), (95), and (38), the Floquet zero eigenfunction for the spatial phase exhibits the following reflection properties (see Figs. 6-8):

$$
\begin{aligned}
& U_{\theta}^{(\mathrm{s})}(-(x-\Phi), y, \Theta)=-U_{\theta}^{(\mathrm{s})}(x-\Phi, y, \Theta), \\
& U_{v}^{(\mathrm{s})}(-(x-\Phi), y, \Theta)=+U_{v}^{(\mathrm{s})}(x-\Phi, y, \Theta), \\
& U_{w}^{(\mathrm{s})}(-(x-\Phi), y, \Theta)=-U_{w}^{(\mathrm{s})}(x-\Phi, y, \Theta) .
\end{aligned}
$$



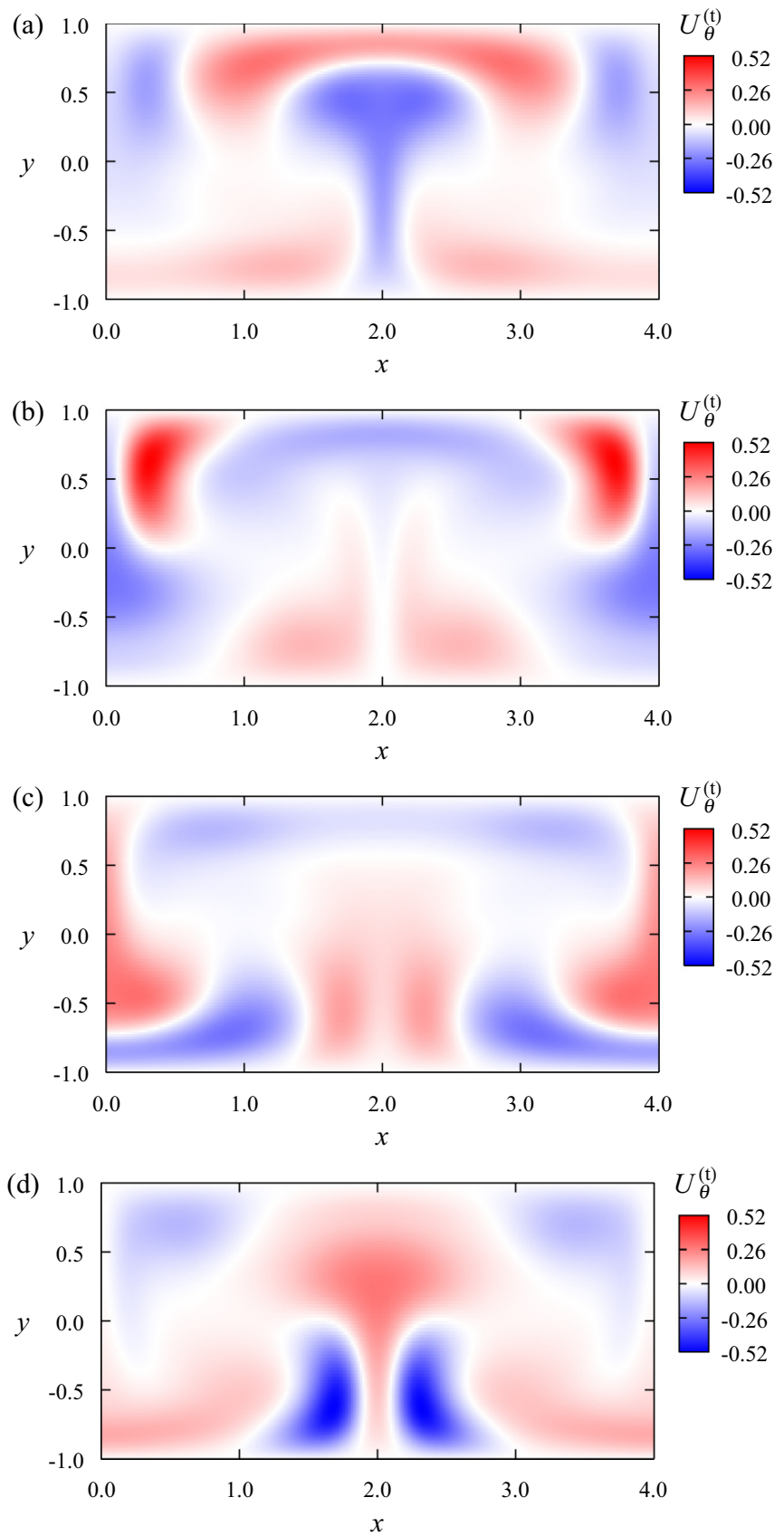

FIG. 9. Snapshots of $U_{\theta}^{(\mathrm{t})}(x-\Phi, y, \Theta)$ with $\Phi=2$ for (a) $\Theta / \pi=0.0$, (b) $\Theta / \pi=0.5$, (c) $\Theta / \pi=1.0$, and (d) $\Theta / \pi=1.5$.

From Eqs. (93), (94), (95), and (39), the Floquet zero eigenfunction for the temporal phase exhibits the following reflection properties (see Figs. 9-11):

$$
\begin{aligned}
& U_{\theta}^{(\mathrm{t})}(-(x-\Phi), y, \Theta)=+U_{\theta}^{(\mathrm{t})}(x-\Phi, y, \Theta), \\
& U_{v}^{(\mathrm{t})}(-(x-\Phi), y, \Theta)=-U_{v}^{(\mathrm{t})}(x-\Phi, y, \Theta), \\
& U_{w}^{(\mathrm{t})}(-(x-\Phi), y, \Theta)=+U_{w}^{(\mathrm{t})}(x-\Phi, y, \Theta) .
\end{aligned}
$$
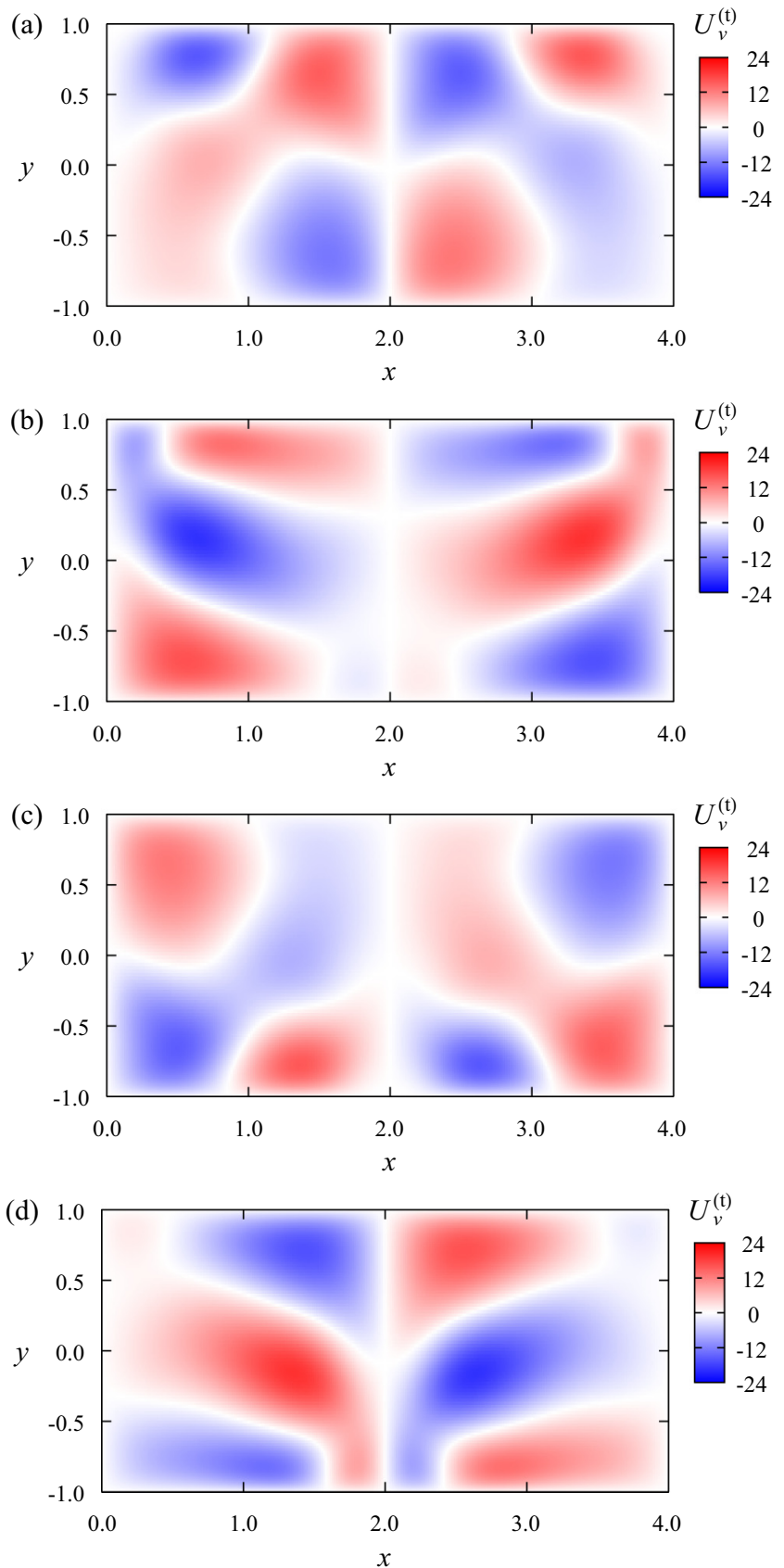

FIG. 10. Snapshots of $U_{v}^{(\mathrm{t})}(x-\Phi, y, \Theta)$ with $\Phi=2$ for (a) $\Theta / \pi=0.0$, (b) $\Theta / \pi=0.5$, (c) $\Theta / \pi=1.0$, and (d) $\Theta / \pi=1.5$.

Similarly, the phase sensitivity function for the spatial phase exhibits the following reflection properties (see Figs. 12-14):

$$
\begin{aligned}
& Z_{\theta}^{(\mathrm{s})}(-(x-\Phi), y, \Theta)=-Z_{\theta}^{(\mathrm{s})}(x-\Phi, y, \Theta), \\
& Z_{v}^{(\mathrm{s})}(-(x-\Phi), y, \Theta)=+Z_{v}^{(\mathrm{s})}(x-\Phi, y, \Theta), \\
& Z_{w}^{(\mathrm{s})}(-(x-\Phi), y, \Theta)=-Z_{w}^{(\mathrm{s})}(x-\Phi, y, \Theta),
\end{aligned}
$$

and the phase sensitivity function for the temporal phase exhibits the following reflection properties (see 
(a)
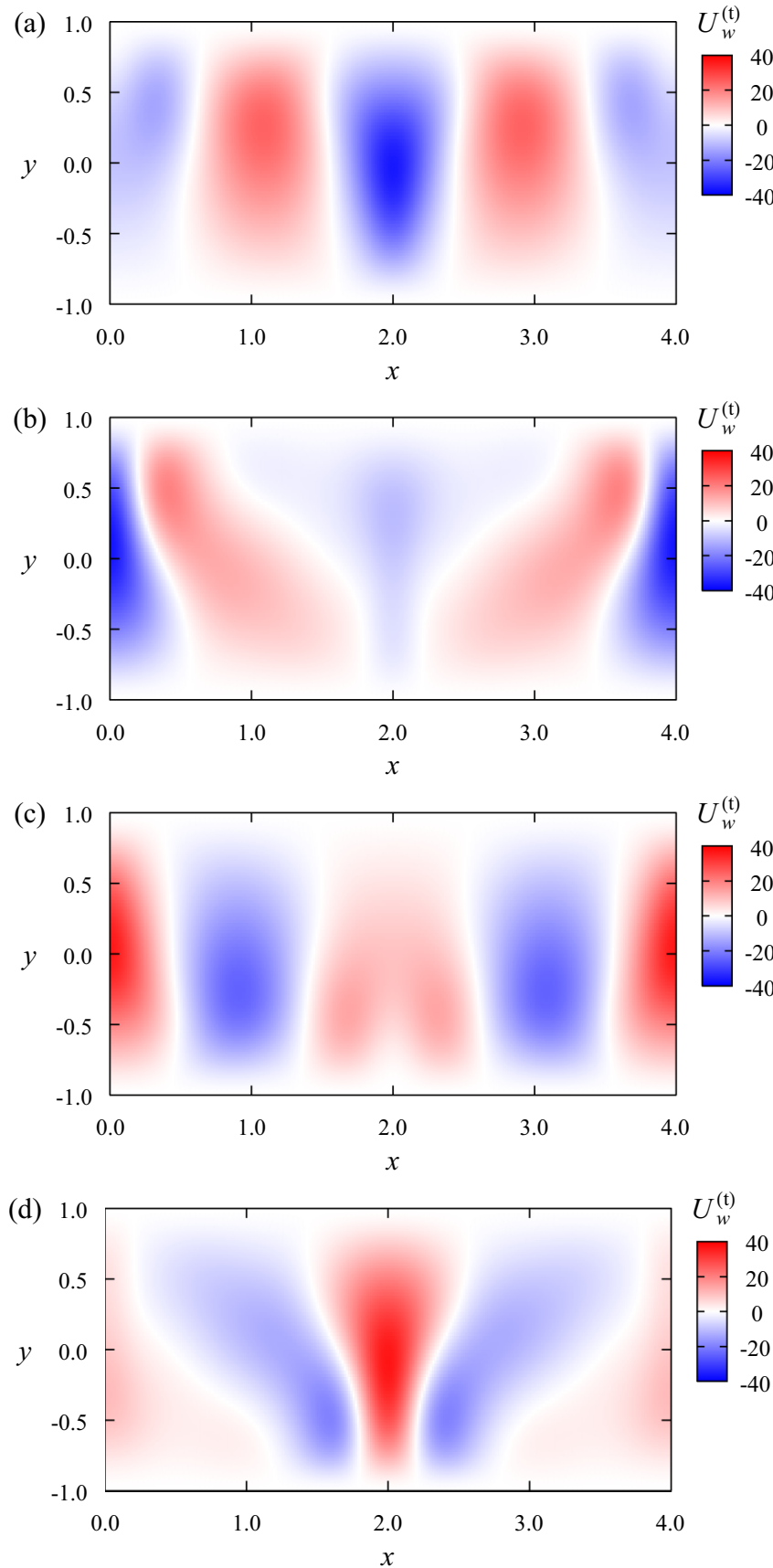

FIG. 11. Snapshots of $U_{w}^{(\mathrm{t})}(x-\Phi, y, \Theta)$ with $\Phi=2$ for (a) $\Theta / \pi=0.0$, (b) $\Theta / \pi=0.5$, (c) $\Theta / \pi=1.0$, and (d) $\Theta / \pi=1.5$.

Figs. 15-17):

$$
\begin{aligned}
& Z_{\theta}^{(\mathrm{t})}(-(x-\Phi), y, \Theta)=+Z_{\theta}^{(\mathrm{t})}(x-\Phi, y, \Theta), \\
& Z_{v}^{(\mathrm{t})}(-(x-\Phi), y, \Theta)=-Z_{v}^{(\mathrm{t})}(x-\Phi, y, \Theta), \\
& Z_{w}^{(\mathrm{t})}(-(x-\Phi), y, \Theta)=+Z_{w}^{(\mathrm{t})}(x-\Phi, y, \Theta) .
\end{aligned}
$$

\section{Phase coupling functions}

In this subsection, we calculate the phase coupling functions obtained in Sec. IIE. As an example, we consider the
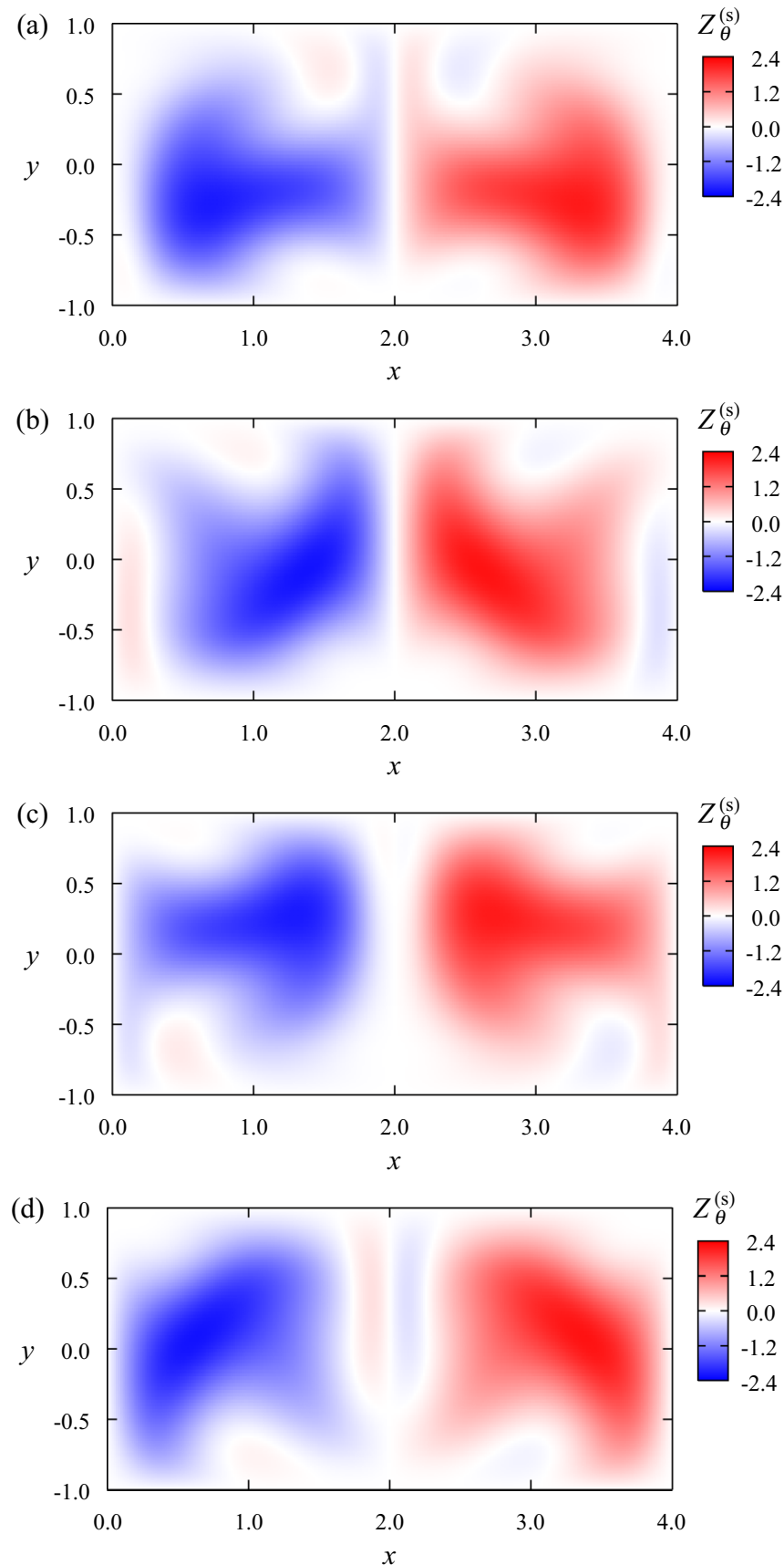

FIG. 12. Snapshots of $Z_{\theta}^{(\mathrm{s})}(x-\Phi, y, \Theta)$ with $\Phi=2$ for (a) $\Theta / \pi=0.0$, (b) $\Theta / \pi=0.5$, (c) $\Theta / \pi=1.0$, and (d) $\Theta / \pi=1.5$.

following thermal coupling:

$$
\mathcal{G}\left[\boldsymbol{X}_{\sigma}, \boldsymbol{X}_{\tau}\right]=\left[0,0,0, \theta_{\tau}(x, y, t)-\theta_{\sigma}(x, y, t)\right]^{\mathrm{T}},
$$

for which the phase coupling functions given by Eqs. (79) and (80) can be written in the following forms:

$$
\begin{aligned}
\Gamma_{\mathrm{s}}(\Phi, \Theta)= & \frac{1}{2 \pi} \int_{0}^{2 \pi} d \lambda \int_{0}^{2 L} d x \int_{-1}^{+1} d y Z_{\theta}^{(\mathrm{s})}(x-\Phi, y, \lambda+\Theta) \\
& \times\left[\theta_{0}(x, y, \lambda)-\theta_{0}(x-\Phi, y, \lambda+\Theta)\right],
\end{aligned}
$$


(a)
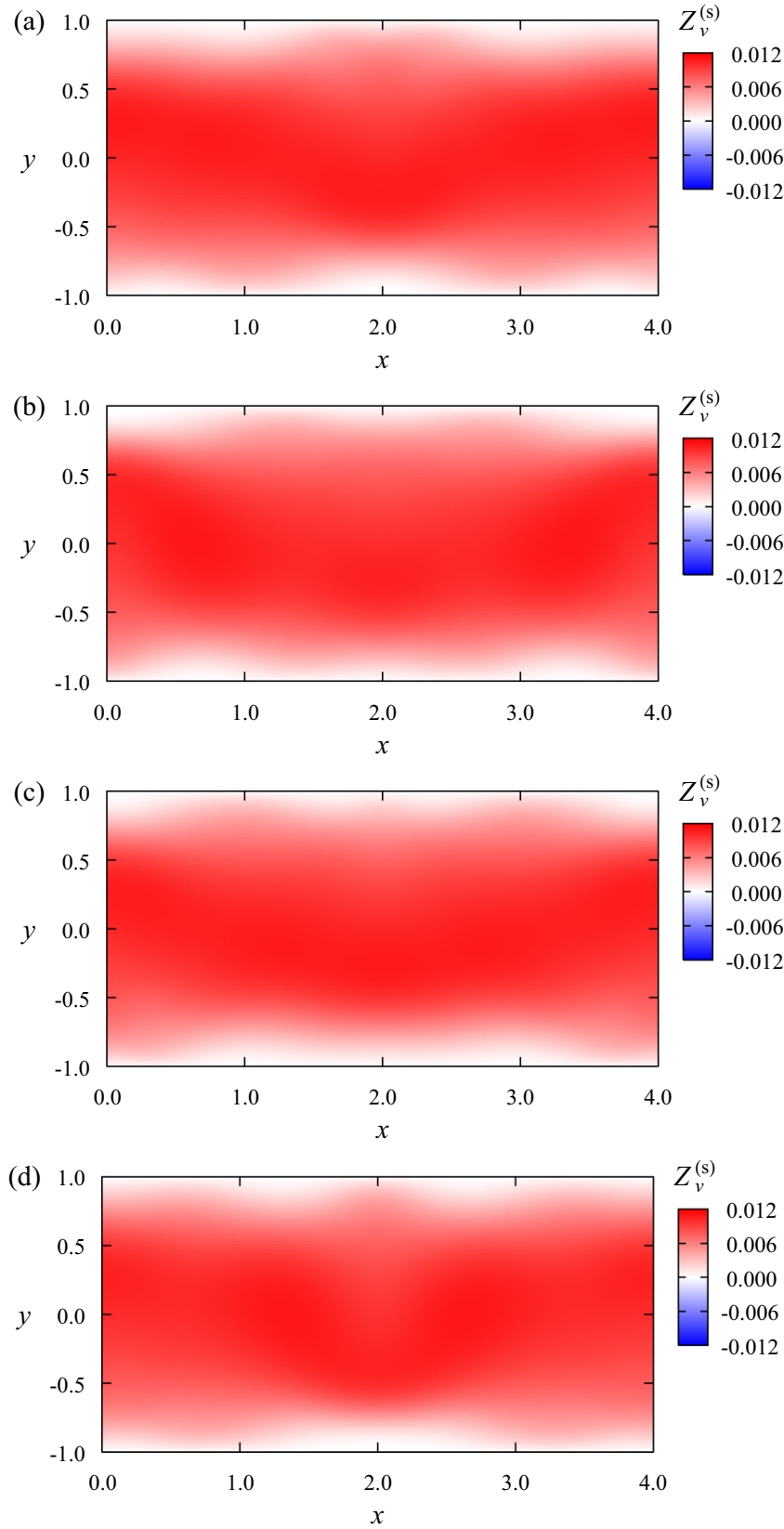

FIG. 13. Snapshots of $Z_{v}^{(\mathrm{s})}(x-\Phi, y, \Theta)$ with $\Phi=2$ for (a) $\Theta / \pi=0.0$, (b) $\Theta / \pi=0.5$, (c) $\Theta / \pi=1.0$, and (d) $\Theta / \pi=1.5$.

$$
\begin{aligned}
\Gamma_{\mathrm{t}}(\Phi, \Theta)= & \frac{1}{2 \pi} \int_{0}^{2 \pi} d \lambda \int_{0}^{2 L} d x \int_{-1}^{+1} d y Z_{\theta}^{(\mathrm{t})}(x-\Phi, y, \lambda+\Theta) \\
& \times\left[\theta_{0}(x, y, \lambda)-\theta_{0}(x-\Phi, y, \lambda+\Theta)\right] .
\end{aligned}
$$

Figures 18 and 19 show the antisymmetric components of the phase coupling functions, $\Gamma_{\mathrm{s}}^{(\mathrm{a})}(\Delta \Phi, \Delta \Theta)$ and $\Gamma_{\mathrm{t}}^{(\mathrm{a})}(\Delta \Phi, \Delta \Theta)$, respectively. As shown in Eqs. (87) and (88), both $\Gamma_{\mathrm{s}}^{(\mathrm{a})}(\Delta \Phi, \Delta \Theta)$ and $\Gamma_{\mathrm{t}}^{(\mathrm{a})}(\Delta \Phi, \Delta \Theta)$ exhibit antisymmetry with respect to the origin, i.e., $\Delta \Phi=\Delta \Theta=0$.

Here, we describe another symmetry of the antisymmetric components of the phase coupling functions in this spatial reflection symmetric case (see Figs. 2, 12, and 15). From Eqs. (93), (117), and (120), the phase coupling functions,
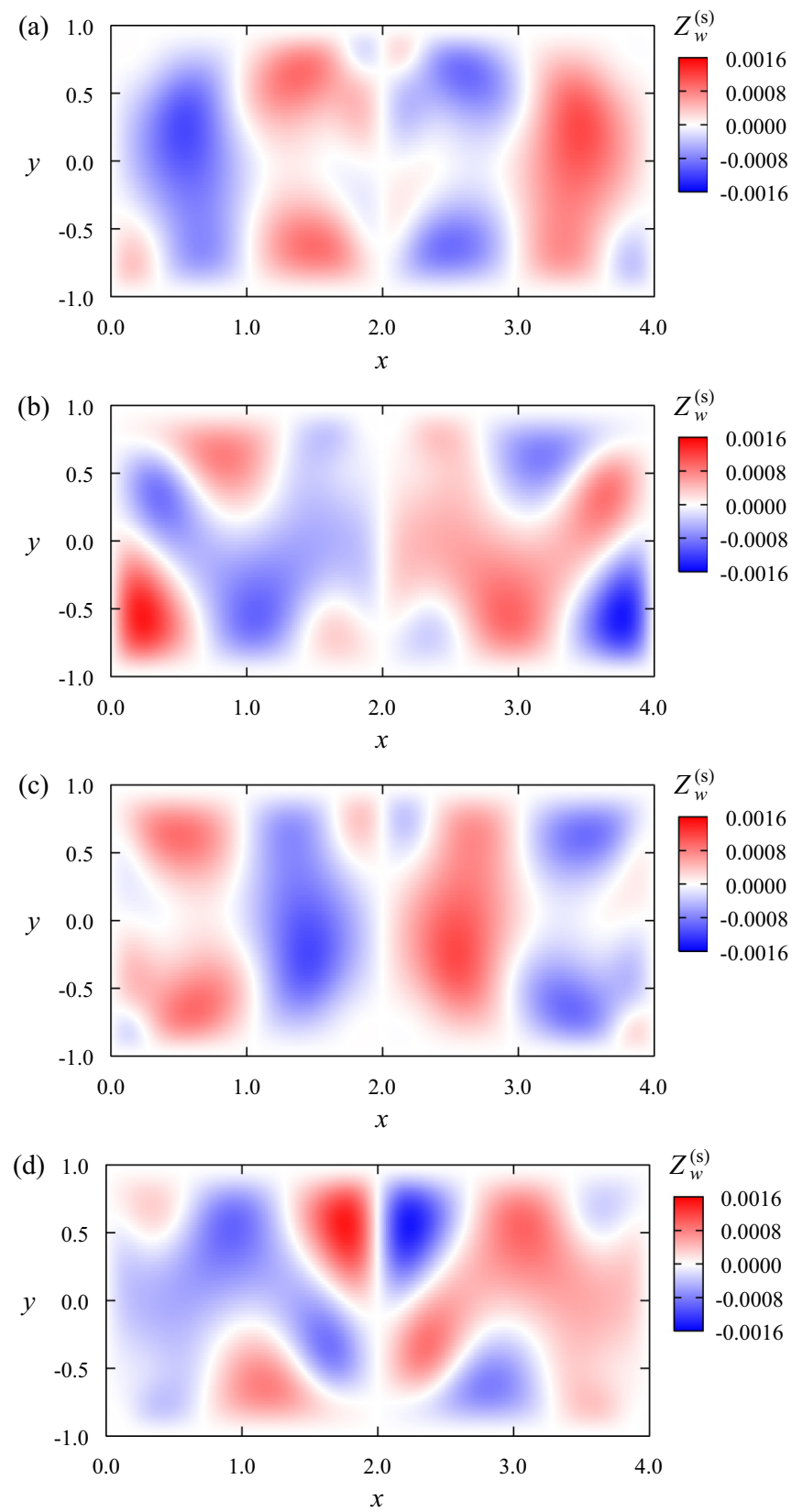

FIG. 14. Snapshots of $Z_{w}^{(\mathrm{s})}(x-\Phi, y, \Theta)$ with $\Phi=2$ for (a) $\Theta / \pi=0.0$, (b) $\Theta / \pi=0.5$, (c) $\Theta / \pi=1.0$, and (d) $\Theta / \pi=1.5$.

$\Gamma_{\mathrm{s}}(\Delta \Phi, \Delta \Theta)$ and $\Gamma_{\mathrm{t}}(\Delta \Phi, \Delta \Theta)$, exhibit the following reflection antisymmetry and reflection symmetry, respectively:

$$
\begin{aligned}
& \Gamma_{\mathrm{s}}(-\Delta \Phi, \Delta \Theta)=-\Gamma_{\mathrm{s}}(\Delta \Phi, \Delta \Theta), \\
& \Gamma_{\mathrm{t}}(-\Delta \Phi, \Delta \Theta)=+\Gamma_{\mathrm{t}}(\Delta \Phi, \Delta \Theta) .
\end{aligned}
$$

Therefore, the antisymmetric components of the phase coupling functions, $\Gamma_{\mathrm{s}}^{(\mathrm{a})}(\Delta \Phi, \Delta \Theta)$ and $\Gamma_{\mathrm{t}}^{(\mathrm{a})}(\Delta \Phi, \Delta \Theta)$, also exhibit the following reflection antisymmetry and reflection symmetry (see Figs. 18 and 19):

$$
\begin{aligned}
& \Gamma_{\mathrm{s}}^{(\mathrm{a})}(-\Delta \Phi, \Delta \Theta)=-\Gamma_{\mathrm{s}}^{(\mathrm{a})}(\Delta \Phi, \Delta \Theta), \\
& \Gamma_{\mathrm{t}}^{(\mathrm{a})}(-\Delta \Phi, \Delta \Theta)=+\Gamma_{\mathrm{t}}^{(\mathrm{a})}(\Delta \Phi, \Delta \Theta) .
\end{aligned}
$$


(a)

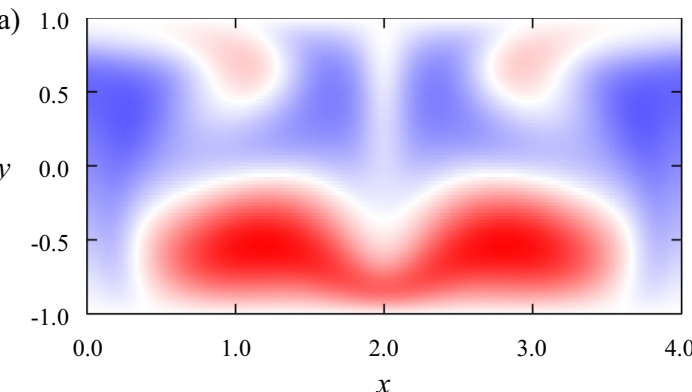

(b)

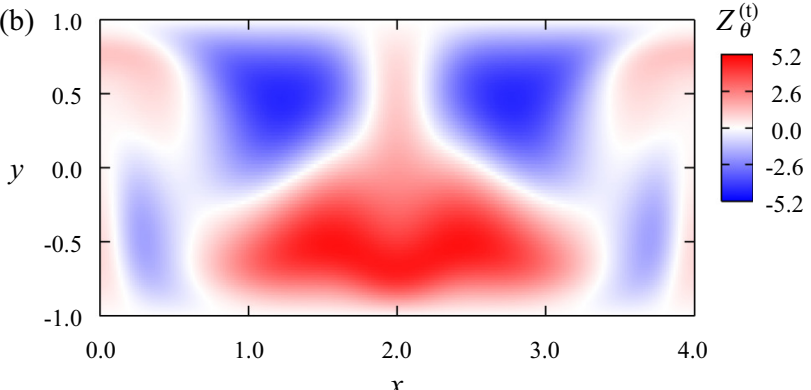

(c)

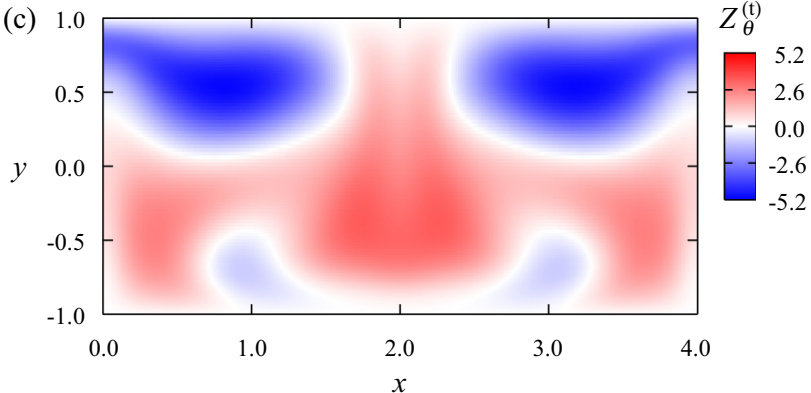

(d)

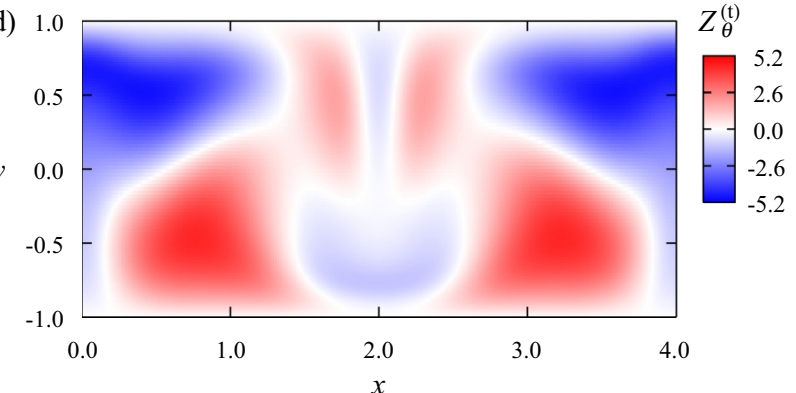

FIG. 15. Snapshots of $Z_{\theta}^{(\mathrm{t})}(x-\Phi, y, \Theta)$ with $\Phi=2$ for (a) $\Theta / \pi=0.0$, (b) $\Theta / \pi=0.5$, (c) $\Theta / \pi=1.0$, and (d) $\Theta / \pi=1.5$.

Further, considering Eqs. (87), (88), (128), and (129), we can also obtain the following properties (see Figs. 18 and 19):

$$
\begin{aligned}
& \Gamma_{\mathrm{s}}^{(\mathrm{a})}(\Delta \Phi,-\Delta \Theta)=+\Gamma_{\mathrm{s}}^{(\mathrm{a})}(\Delta \Phi, \Delta \Theta), \\
& \Gamma_{\mathrm{t}}^{(\mathrm{a})}(\Delta \Phi,-\Delta \Theta)=-\Gamma_{\mathrm{t}}^{(\mathrm{a})}(\Delta \Phi, \Delta \Theta) .
\end{aligned}
$$

It is evident from the above properties that the investigation of only the following region: $(\Delta \Phi / L, \Delta \Theta / \pi) \in[0,1] \times$ $[0,1]$ is sufficient. Figure 20 shows the nullclines, equilibrium points, and typical orbits of the spatiotemporal phase differences in this region. The typical orbits were obtained from the numerical solutions of Eqs. (83) and (84). As
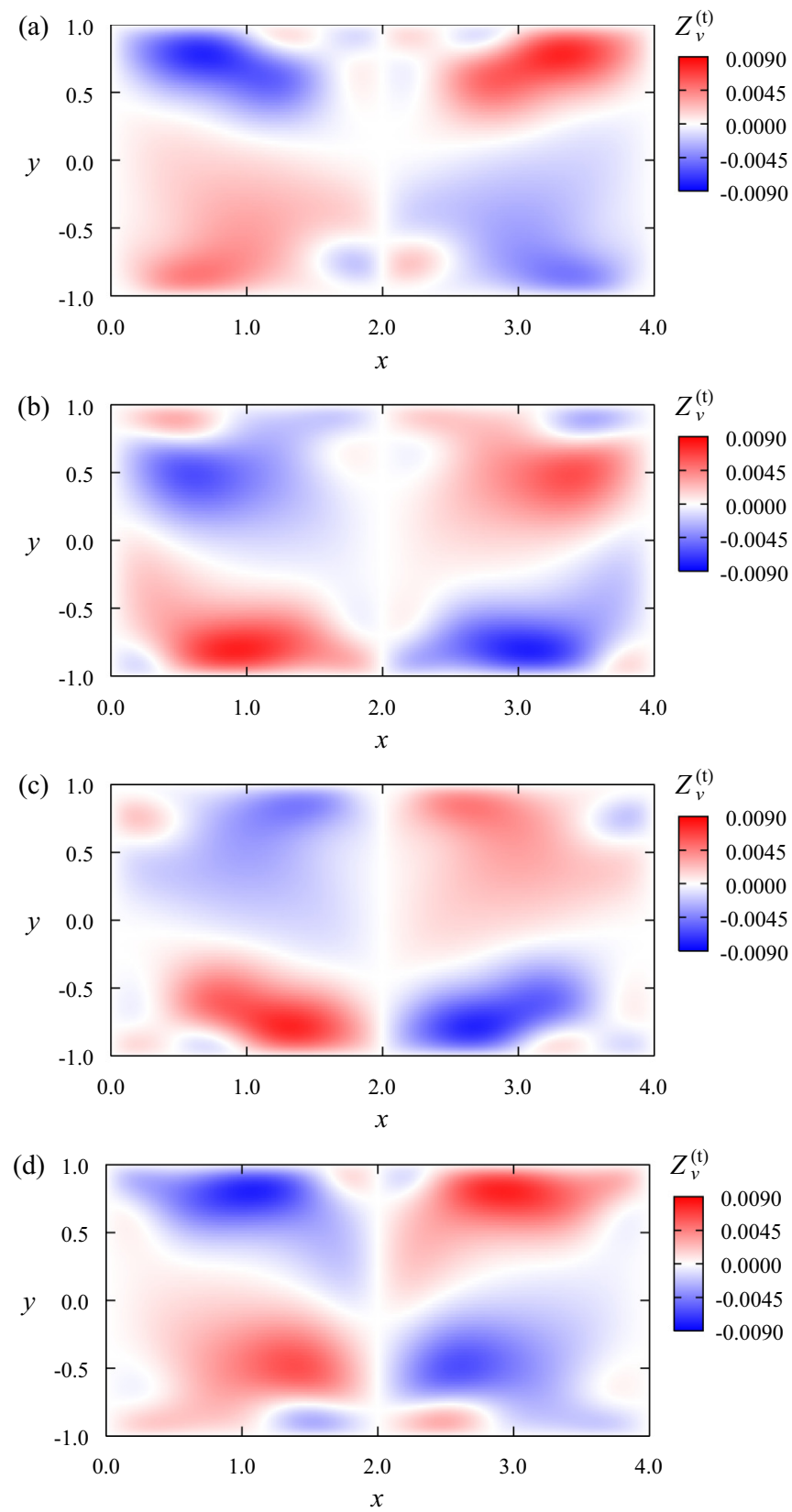

FIG. 16. Snapshots of $Z_{v}^{(\mathrm{t})}(x-\Phi, y, \Theta)$ with $\Phi=2$ for (a) $\Theta / \pi=0.0$, (b) $\Theta / \pi=0.5$, (c) $\Theta / \pi=1.0$, and (d) $\Theta / \pi=1.5$.

seen in Fig. 20, the spatial and temporal in-phase state, i.e., $(\Delta \Phi / L, \Delta \Theta / \pi)=(0,0)$, is globally stable under the phase reduction approximation. The spatial phase difference $\Delta \Phi$ monotonously decreases to zero, whereas the temporal phase difference $\Delta \Theta$ first increases slightly and then becomes zero.

\section{Direct numerical simulations}

In this subsection, we compare the time evolution of spatiotemporal phase differences between the theoretical values obtained in Sec. III C (shown in Fig. 20) and the direct numerical simulations of two weakly coupled systems exhibiting oscillatory thermal convection described by Eqs. (67) and (123). 

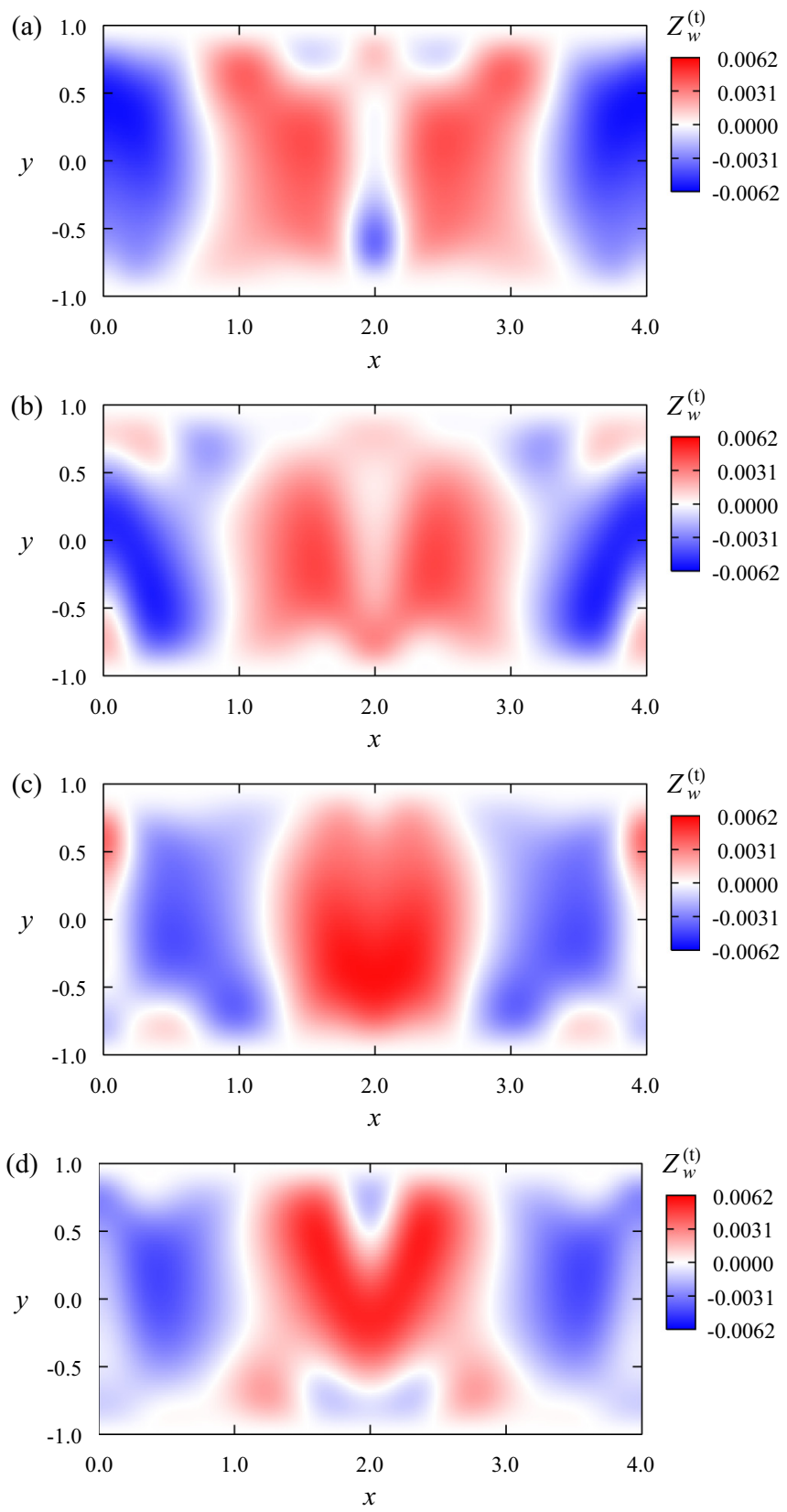

FIG. 17. Snapshots of $Z_{w}^{(\mathrm{t})}(x-\Phi, y, \Theta)$ with $\Phi=2$ for (a) $\Theta / \pi=0.0$, (b) $\Theta / \pi=0.5$, (c) $\Theta / \pi=1.0$, and (d) $\Theta / \pi=1.5$.

Figures 21 and 22 show comparisons of the time evolution of the spatial and temporal phase differences, respectively. Figure 23 shows comparisons of the orbits of the spatiotemporal phase differences. There is an excellent agreement between the theoretical and simulation results.

\section{CONCLUDING REMARKS}

In this paper, we formulated a theory for the phase reduction of traveling and oscillating thermal convection in incompressible Navier-Stokes flow systems, namely, limit-torus solutions to partial differential algebraic equations. In particular, we derived the spatiotemporal phase sensitivity functions for the thermal convection and analyzed the spatiotemporal phase

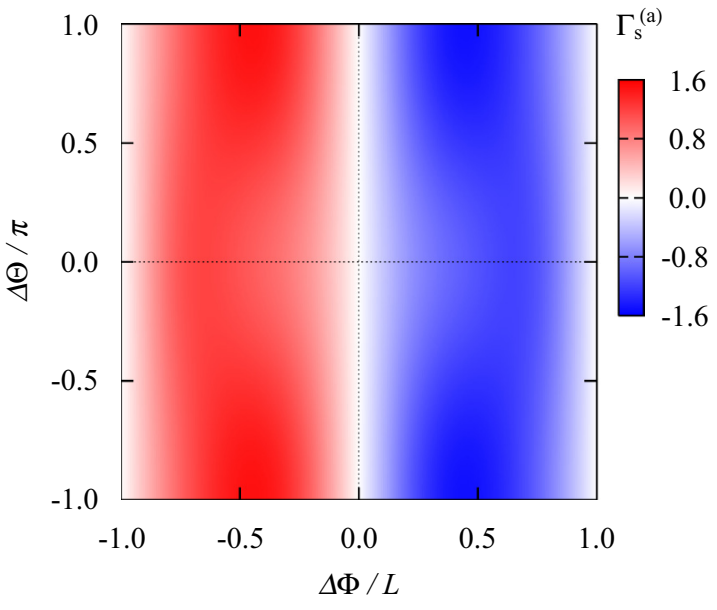

FIG. 18. Antisymmetric component of the phase coupling function for the spatial phase, $\Gamma_{\mathrm{s}}^{(\mathrm{a})}(\Delta \Phi, \Delta \Theta)$.

synchronization between a pair of weakly coupled systems exhibiting the thermal convection. The phase reduction theory enabled us to prove the global stability of the spatial and temporal in-phase state.

The important implications of this study are as follows. First, as observed in Eqs. (109) and (110), the phase sensitivity functions of fluid velocity fields for spatial and temporal phases are divergence-free for incompressible Navier-Stokes flow systems. This is true for both limit-torus and limit-cycle solutions. Further, the temperature field is not essential for this divergence-free property. Therefore, the phase sensitivity function of a fluid velocity field (for the temporal phase) should be divergence-free for periodic flows such as Kármán's vortex street in incompressible Navier-Stokes flow systems [50,51]. The phase reduction theory formulated in this paper will prove to be useful for analyzing the periodic flows.

Second, while an incompressible Navier-Stokes flow system is a representative example of partial differential algebraic equation, another typical example is a partial differential equation with a constraint that represents some conservation. In Ref. [26], we formulated a theory for the phase reduction

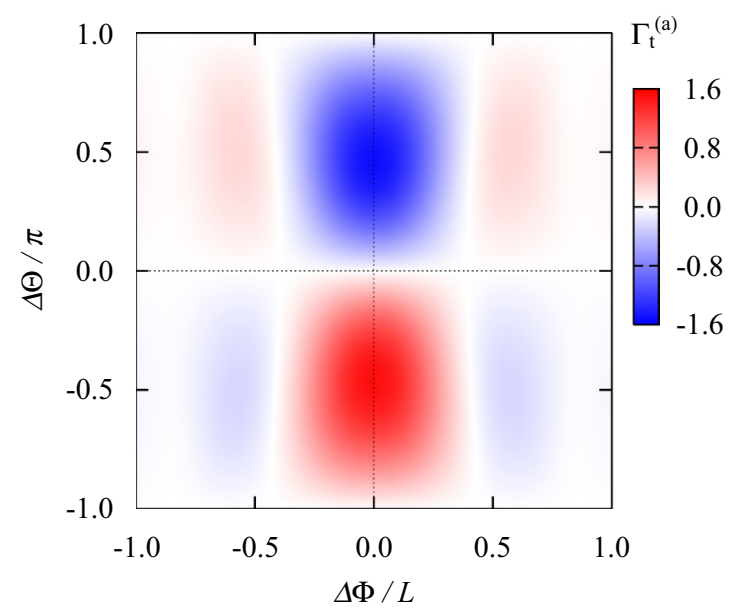

FIG. 19. Antisymmetric component of the phase coupling function for the temporal phase, $\Gamma_{\mathrm{t}}^{(\mathrm{a})}(\Delta \Phi, \Delta \Theta)$. 


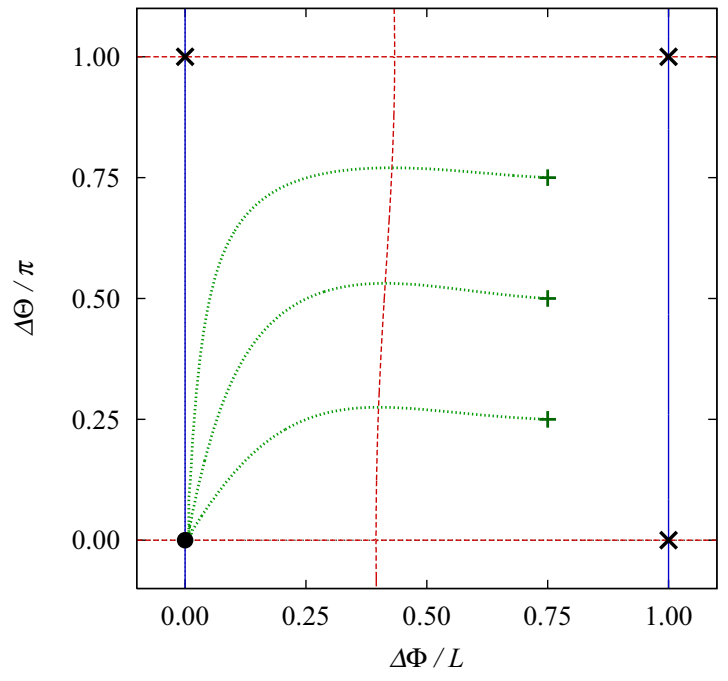

FIG. 20. Nullclines, equilibrium points, and typical orbits of the spatiotemporal phase differences in the following region: $(\Delta \Phi / L, \Delta \Theta / \pi) \in[0,1] \times[0,1]$. The solid (blue) and broken (red) lines indicate the nullclines of $\Gamma_{\mathrm{s}}^{(\mathrm{a})}(\Delta \Phi, \Delta \Theta)$ and $\Gamma_{\mathrm{t}}^{(\mathrm{a})}(\Delta \Phi, \Delta \Theta)$, respectively. The filled circle $(\bullet)$ indicates the stable equilibrium point $(\Delta \Phi / L, \Delta \Theta / \pi)=(0,0)$, whereas the times signs $(\times)$ indicate the unstable equilibrium points $(\Delta \Phi / L, \Delta \Theta / \pi)=(0,1),(1,0)$, and $(1,1)$. The dotted (green) lines indicate the typical orbits of the spatiotemporal phase differences whose initial values indicated by the plus signs $(+)$ are $(\Delta \Phi / L, \Delta \Theta / \pi)=(0.75,0.25),(0.75,0.50)$, and $(0.75,0.75)$.

of a beating flagellum represented by a limit-cycle solution of a partial differential equation model proposed by Goldstein et al. in Ref. [52]. Although this model does not enforce filament length conservation beyond linear order, a model that enforces the length conservation is proposed by De Canio et al. in Ref. [53]. We can use the present idea to formulate a theory for the phase reduction of a beating flagellum

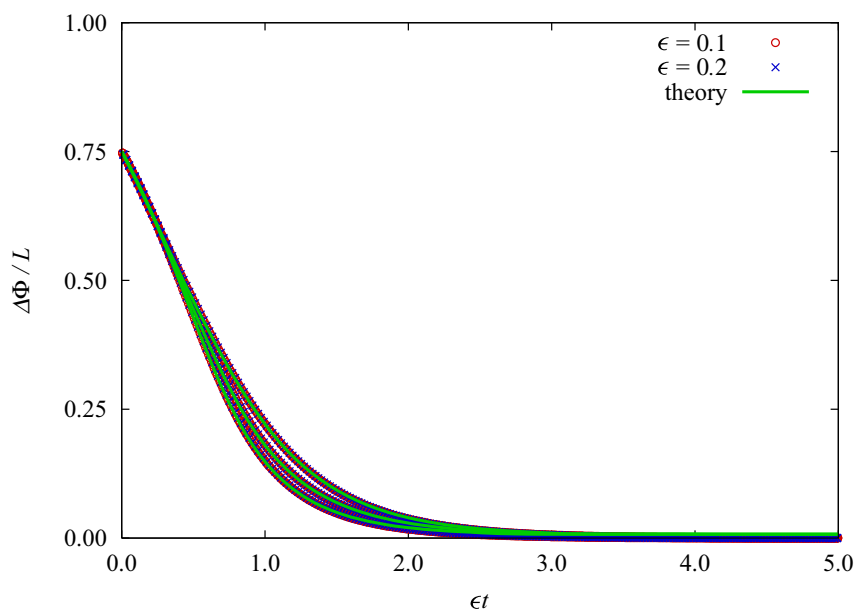

FIG. 21. Comparisons of the time evolution of the spatial phase differences, $\Delta \Phi / L$ vs $\epsilon t$, obtained by direct numerical simulations ( $\epsilon=0.1$ and 0.2$)$ and the theoretical formulation (theory). The initial values are $(\Delta \Phi / L, \Delta \Theta / \pi)=(0.75,0.25),(0.75,0.50)$, and $(0.75,0.75)$.

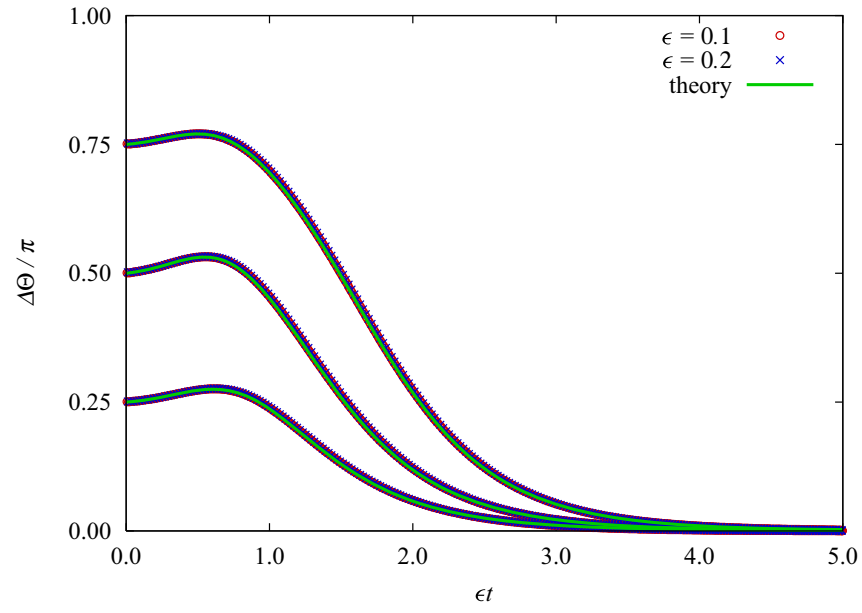

FIG. 22. Comparisons of the time evolution of the temporal phase differences, $\Delta \Theta / \pi$ vs $\epsilon t$, obtained by direct numerical simulations ( $\epsilon=0.1$ and 0.2$)$ and the theoretical formulation (theory). The initial values are $(\Delta \Phi / L, \Delta \Theta / \pi)=(0.75,0.25),(0.75,0.50)$, and $(0.75,0.75)$.

represented by a limit-cycle solution to a partial differential equation constrained with filament length conservation.

Third, we have presented three types of phase reduction methods and corresponding solutions for partial differential equations with and without constraints for the field $\boldsymbol{X}(x, y, t)$. (i) A traveling solution: $\boldsymbol{X}(x, y, t)=$ $\boldsymbol{X}_{0}(x-\Phi(t), y)$ with $\dot{\Phi}(t)=c$. (ii) An oscillating solution: $\boldsymbol{X}(x, y, t)=\boldsymbol{X}_{0}(x, y, \Theta(t))$ with $\dot{\Theta}(t)=\omega$. (iii) A traveling and oscillating solution: $\boldsymbol{X}(x, y, t)=\boldsymbol{X}_{0}(x-\Phi(t), y, \Theta(t))$ with $\dot{\Phi}(t)=c$ and $\dot{\Theta}(t)=\omega$. The phase reduction method for type (i) has been developed in Refs. [54-57] for traveling solutions to nonlinear Fokker-Planck equations; this method is closely related to the phase reduction method for spatially periodic patterns in that the phase mode is associated with spatial translational symmetry breaking [58-63] (see also Refs. [2,27-30,64-66]). ${ }^{2}$ As mentioned in Refs. [20,22,24,46], type (i) can be considered as a special case of type (ii). Type (ii) can be considered as a limit-cycle solution that exhibits only one phase mode, whereas type (iii) can be considered as a limit-torus solution that exhibits two phase modes. The phase reduction methods developed in this paper and in Ref. [46] belong to type (iii), and they can be considered as a generalization of our phase reduction methods for type (ii) developed in Refs. [20-26].

Fourth, we have investigated the spatial reflection symmetry of rhythmic patterns. The traveling velocity $c$ is zero (nonzero) when the rhythmic pattern does not break (breaks) the spatial reflection symmetry. The traveling velocity of the thermal convection studied in this paper is zero; however, the

\footnotetext{
${ }^{2}$ When there exists a conserved quantity in a system, the conserved quantity should also be taken into account in the theory (see Refs. $[62,63,66])$. For example, in the case of stress-free boundary conditions instead of no-slip boundary conditions, the horizontal mean flow is a conserved quantity associated with the Galilean invariance of the Navier-Stokes equation. Consequently, the phase dynamics becomes second-order in time (see Refs. [62,63]).
} 


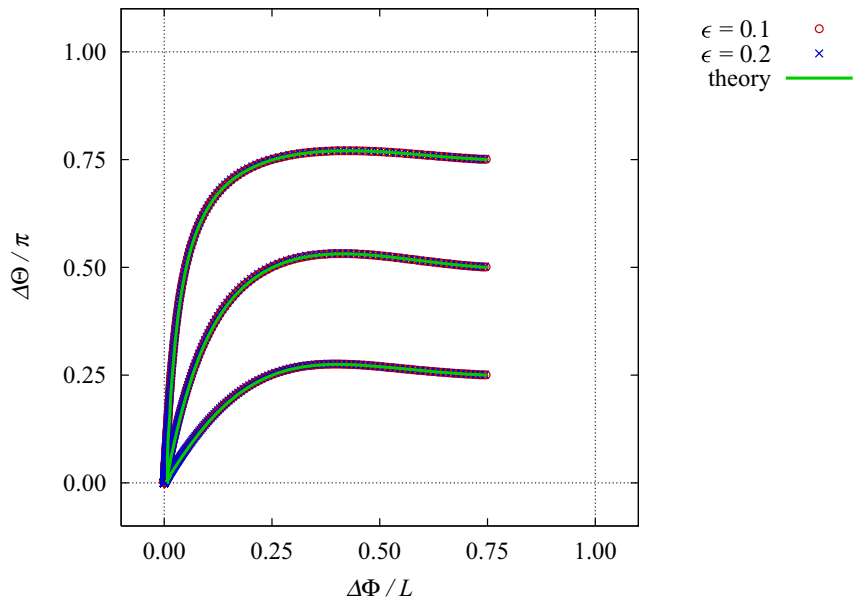

FIG. 23. Comparisons of the orbits of the spatiotemporal phase differences, $\Delta \Theta / \pi$ vs $\Delta \Phi / L$, obtained with direct numerical simulations $(\epsilon=0.1$ and 0.2$)$ and the theoretical formulation (theory). The initial values are $(\Delta \Phi / L, \Delta \Theta / \pi)=(0.75,0.25),(0.75,0.50)$, and $(0.75,0.75)$.

phase reduction method itself is applicable for the case of nonzero traveling velocity. If the reflection symmetry of limittorus solution is broken, the corresponding symmetry of phase sensitivity and phase coupling functions are also broken.

Fifth, our phase reduction approach has wide applicability. Apart from thermal convection described in this paper, this approach will be applied to other phenomena described by limit-torus solutions, i.e., traveling and oscillating solutions. For example, traveling and oscillating localized convection (i.e., a traveling breather) in a binary fluid system (see, e.g., Ref. [67]) and rotating and oscillating convection (i.e., amplitude vacillation [40-42]) in a rotating fluid annulus system (see, e.g., Ref. [68,69]). Further, the numerical methods based on Refs. [70-72], which are summarized in Appendixes $\mathrm{B}$ and $\mathrm{C}$, can be generalized for rotating flows $[73,74]$. In addition, as mentioned in Ref. [46], similar phase reduction methods can be developed for oscillating spots of zero traveling velocity (see, e.g., Ref. [75]) or traveling breathers of nonzero traveling velocity (see, e.g., Ref. [76]) in reaction-diffusion systems.

Finally, the proposed method can be considered as a generalized phase reduction method, which can be elucidated as follows. The first point is the generalization from limit-cycle solutions (i.e., oscillating solutions) to limit-torus solutions (i.e., traveling and oscillating solutions). The second point is the generalization from ordinary differential equations to partial differential equations (e.g., reaction-diffusion equations [24,25] and beating flagella [26]), partial differential integral equations (e.g., nonlinear Fokker-Planck equations $[20,21]$ and Hele-Shaw cells $[22,23,46])$, and partial differential algebraic equations (e.g., incompressible Navier-Stokes equations including rotating fluid annuli). That is, in this paper, we developed the phase reduction method for limit-torus solutions to partial differential algebraic equations. The future scope of this study involves the development of an advanced theory, which can be applied to rotating fluid annulus [43-45] introduced in Sec. I. We believe that such a study will provide novel insights into meteorology (see, e.g., Refs. [77,78]).

\section{ACKNOWLEDGMENTS}

This work was supported by JSPS (Japan) KAKENHI Grants No. JP18H03205, No. JP17H03279, and No. JP16K17769. This work was also supported by the Research Institute for Mathematical Sciences, an International Joint Usage/Research Center located in Kyoto University.

\section{APPENDIX A: EXPLICIT FORMS OF ADJOINT SYSTEMS}

In this Appendix, we describe the details of the derivation of the adjoint operator $\hat{\mathcal{L}}^{*}(x-\Phi, y, \Theta)$ including the adjoint boundary conditions encountered in Sec. IIB (see also, e.g., Refs. [79-81] for mathematical terms). Here, we denote the components of $\boldsymbol{u}(x-\Phi, y, \Theta)$ as

$$
\boldsymbol{u}(x-\Phi, y, \Theta)=\left(p_{1}, v_{1}, w_{1}, \theta_{1}\right)^{\mathrm{T}}, \quad \boldsymbol{v}_{1}=\left(v_{1}, w_{1}\right)^{\mathrm{T}} .
$$

Further, we also denote the components of $\boldsymbol{u}^{*}(x-\Phi, y, \Theta)$ as

$$
\boldsymbol{u}^{*}(x-\Phi, y, \Theta)=\left(p_{1}^{*}, v_{1}^{*}, w_{1}^{*}, \theta_{1}^{*}\right), \quad \boldsymbol{v}_{1}^{*}=\left(v_{1}^{*}, w_{1}^{*}\right) .
$$

From Eqs. (27) and (28), the linear operator $\hat{\mathcal{L}}(x-\Phi, y, \Theta)$ is given by

$$
\hat{\mathcal{L}}(x-\Phi, y, \Theta) \boldsymbol{u}(x-\Phi, y, \Theta)=\left[\hat{\mathcal{J}}(x-\Phi, y, \Theta)+\hat{D} \nabla^{2}+c \hat{M} \frac{\partial}{\partial x}-\omega \hat{M} \frac{\partial}{\partial \Theta}\right] \boldsymbol{u}(x-\Phi, y, \Theta) .
$$

From Eqs. (32) and (33), the adjoint operator $\hat{\mathcal{L}}^{*}(x-\Phi, y, \Theta)$ is given by

$$
\hat{\mathcal{L}}^{*}(x-\Phi, y, \Theta) \boldsymbol{u}^{*}(x-\Phi, y, \Theta)=\left[\hat{\mathcal{J}}^{*}(x-\Phi, y, \Theta)+\hat{D}^{\mathrm{T}} \nabla^{2}-c \hat{M}^{\mathrm{T}} \frac{\partial}{\partial x}+\omega \hat{M}^{\mathrm{T}} \frac{\partial}{\partial \Theta}\right] \boldsymbol{u}^{*}(x-\Phi, y, \Theta) .
$$

Here, the two singular diagonal matrices defined in Eq. (18) are symmetric, i.e.,

$$
\hat{M}=\hat{M}^{\mathrm{T}}=\left(\begin{array}{llll}
0 & 0 & 0 & 0 \\
0 & 1 & 0 & 0 \\
0 & 0 & 1 & 0 \\
0 & 0 & 0 & 1
\end{array}\right), \quad \hat{D}=\hat{D}^{\mathrm{T}}=\left(\begin{array}{cccc}
0 & 0 & 0 & 0 \\
0 & \operatorname{Pr} & 0 & 0 \\
0 & 0 & \operatorname{Pr} & 0 \\
0 & 0 & 0 & 1
\end{array}\right) \text {. }
$$


For readability, Eq. (17) is reproduced as

$$
\mathcal{F}[\boldsymbol{X}]=\left\{\begin{array}{l}
\boldsymbol{\nabla} \cdot \boldsymbol{v} \\
-\boldsymbol{v} \cdot \nabla v-\partial_{x} p \\
-\boldsymbol{v} \cdot \nabla w-\partial_{y} p+\operatorname{Pr} \operatorname{Ra} \theta \\
-\boldsymbol{v} \cdot \nabla \theta+w
\end{array}\right.
$$

The linearization of Eq. (A6) around the limit-torus solution $\boldsymbol{X}_{0}(x-\Phi, y, \Theta)$ [denoted by Eq. (23)] implies

$$
\hat{\mathcal{J}} \boldsymbol{u}=\left\{\begin{array}{l}
\boldsymbol{\nabla} \cdot \boldsymbol{v}_{1}, \\
-\boldsymbol{v}_{1} \cdot \nabla v_{0}-\boldsymbol{v}_{0} \cdot \nabla v_{1}-\partial_{x} p_{1}, \\
-\boldsymbol{v}_{1} \cdot \nabla w_{0}-\boldsymbol{v}_{0} \cdot \nabla w_{1}-\partial_{y} p_{1}+\operatorname{Pr} \operatorname{Ra} \theta_{1}, \\
-\boldsymbol{v}_{1} \cdot \nabla \theta_{0}-\boldsymbol{v}_{0} \cdot \nabla \theta_{1}+w_{1},
\end{array}\right.
$$

which can also be written in the following form:

$$
\hat{\mathcal{J}} \boldsymbol{u}=\left\{\begin{array}{l}
\partial_{x} v_{1}+\partial_{y} w_{1}, \\
-v_{1} \partial_{x} v_{0}-w_{1} \partial_{y} v_{0}-v_{0} \partial_{x} v_{1}-w_{0} \partial_{y} v_{1}-\partial_{x} p_{1}, \\
-v_{1} \partial_{x} w_{0}-w_{1} \partial_{y} w_{0}-v_{0} \partial_{x} w_{1}-w_{0} \partial_{y} w_{1}-\partial_{y} p_{1}+\operatorname{Pr} \operatorname{Ra} \theta_{1}, \\
-v_{1} \partial_{x} \theta_{0}-w_{1} \partial_{y} \theta_{0}-v_{0} \partial_{x} \theta_{1}-w_{0} \partial_{y} \theta_{1}+w_{1} .
\end{array}\right.
$$

Therefore, the explicit form of $\hat{\mathcal{J}}^{*}(x-\Phi, y, \Theta) \boldsymbol{u}^{*}(x-\Phi, y, \Theta)$ is

$$
\hat{\mathcal{J}}^{*} \boldsymbol{u}^{*}=\left\{\begin{array}{l}
\partial_{x} v_{1}^{*}+\partial_{y} w_{1}^{*}, \\
\partial_{x}\left[v_{1}^{*} v_{0}\right]+\partial_{y}\left[v_{1}^{*} w_{0}\right]-v_{1}^{*} \partial_{x} v_{0}-w_{1}^{*} \partial_{x} w_{0}-\partial_{x} p_{1}^{*}-\theta_{1}^{*} \partial_{x} \theta_{0}, \\
\partial_{x}\left[w_{1}^{*} v_{0}\right]+\partial_{y}\left[w_{1}^{*} w_{0}\right]-v_{1}^{*} \partial_{y} v_{0}-w_{1}^{*} \partial_{y} w_{0}-\partial_{y} p_{1}^{*}-\theta_{1}^{*} \partial_{y} \theta_{0}+\theta_{1}^{*}, \\
\partial_{x}\left[\theta_{1}^{*} v_{0}\right]+\partial_{y}\left[\theta_{1}^{*} w_{0}\right]+\operatorname{Pr} \operatorname{Ra} w_{1}^{*},
\end{array}\right.
$$

which can also be expressed as

$$
\hat{\mathcal{J}}^{*} \boldsymbol{u}^{*}=\left\{\begin{array}{l}
\boldsymbol{\nabla} \cdot \boldsymbol{v}_{1}^{*}, \\
\boldsymbol{\nabla} \cdot\left[v_{1}^{*} \boldsymbol{v}_{0}\right]-\boldsymbol{v}_{1}^{*} \cdot \partial_{x} \boldsymbol{v}_{0}-\partial_{x} p_{1}^{*}-\theta_{1}^{*} \partial_{x} \theta_{0}, \\
\nabla \cdot\left[w_{1}^{*} \boldsymbol{v}_{0}\right]-\boldsymbol{v}_{1}^{*} \cdot \partial_{y} \boldsymbol{v}_{0}-\partial_{y} p_{1}^{*}-\theta_{1}^{*} \partial_{y} \theta_{0}+\theta_{1}^{*}, \\
\nabla \cdot\left[\theta_{1}^{*} \boldsymbol{v}_{0}\right]+\operatorname{Pr} \operatorname{Ra} w_{1}^{*} .
\end{array}\right.
$$

The boundary conditions on $y$ for $\boldsymbol{u}(x-\Phi, y, \Theta)$ are

$$
\begin{aligned}
& \left.v_{1}(x-\Phi, y, \Theta)\right|_{y= \pm 1}=0, \\
& \left.w_{1}(x-\Phi, y, \Theta)\right|_{y= \pm 1}=0, \\
& \left.\theta_{1}(x-\Phi, y, \Theta)\right|_{y= \pm 1}=0 .
\end{aligned}
$$

Similarly, the adjoint boundary conditions on $y$ for $\boldsymbol{u}^{*}(x-\Phi, y, \Theta)$ are

$$
\begin{aligned}
& \left.v_{1}^{*}(x-\Phi, y, \Theta)\right|_{y= \pm 1}=0, \\
& \left.w_{1}^{*}(x-\Phi, y, \Theta)\right|_{y= \pm 1}=0, \\
& \left.\theta_{1}^{*}(x-\Phi, y, \Theta)\right|_{y= \pm 1}=0 .
\end{aligned}
$$

In fact, under these adjoint boundary conditions, the bilinear concomitant on $y$ becomes zero as follows:

$$
\begin{aligned}
\mathcal{S}\left[\boldsymbol{u}^{*}(x-\Phi, y, \Theta), \boldsymbol{u}(x-\Phi, y, \Theta)\right] \\
=+\frac{1}{2 \pi} \int_{0}^{2 \pi} d \Theta \int_{0}^{2 L} d x\left[p_{1}^{*} w_{1}\right]_{y=-1}^{y=+1}-\frac{1}{2 \pi} \int_{0}^{2 \pi} d \Theta \int_{0}^{2 L} d x\left[w_{1}^{*} p_{1}\right]_{y=-1}^{y=+1}-\frac{1}{2 \pi} \int_{0}^{2 \pi} d \Theta \int_{0}^{2 L} d x\left[v_{1}^{*} w_{0} v_{1}\right]_{y=-1}^{y=+1} \\
\quad-\frac{1}{2 \pi} \int_{0}^{2 \pi} d \Theta \int_{0}^{2 L} d x\left[w_{1}^{*} w_{0} w_{1}\right]_{y=-1}^{y=+1}-\frac{1}{2 \pi} \int_{0}^{2 \pi} d \Theta \int_{0}^{2 L} d x\left[\theta_{1}^{*} w_{0} \theta_{1}\right]_{y=-1}^{y=+1}+\frac{\operatorname{Pr}}{2 \pi} \int_{0}^{2 \pi} d \Theta \int_{0}^{2 L} d x\left[v_{1}^{*} \frac{\partial v_{1}}{\partial y}\right]_{y=-1}^{y=+1} \\
\quad+\frac{\operatorname{Pr}}{2 \pi} \int_{0}^{2 \pi} d \Theta \int_{0}^{2 L} d x\left[w_{1}^{*} \frac{\partial w_{1}}{\partial y}\right]_{y=-1}^{y=+1}+\frac{1}{2 \pi} \int_{0}^{2 \pi} d \Theta \int_{0}^{2 L} d x\left[\theta_{1}^{*} \frac{\partial \theta_{1}}{\partial y}\right]_{y=-1}^{y=+1}-\frac{\operatorname{Pr}}{2 \pi} \int_{0}^{2 \pi} d \Theta \int_{0}^{2 L} d x\left[\frac{\partial v_{1}^{*}}{\partial y} v_{1}\right]_{y=-1}^{y=+1} \\
\quad-\frac{\operatorname{Pr}}{2 \pi} \int_{0}^{2 \pi} d \Theta \int_{0}^{2 L} d x\left[\frac{\partial w_{1}^{*}}{\partial y} w_{1}\right]_{y=-1}^{y=+1}-\frac{1}{2 \pi} \int_{0}^{2 \pi} d \Theta \int_{0}^{2 L} d x\left[\frac{\partial \theta_{1}^{*}}{\partial y} \theta_{1}\right]_{y=-1}^{y=+1}=0 .
\end{aligned}
$$


The bilinear concomitant on both $x$ and $\Theta$ becomes zero, owing to the periodicity with respect to both $x$ and $\Theta$. Finally, from Eqs. (A5) and (A10), Eq. (44) can be explicitly described as

$$
\begin{gathered}
0=\nabla \cdot \boldsymbol{v}^{*}, \\
\frac{\partial}{\partial s} v^{*}(x-\Phi, y,-\omega s)=\nabla \cdot\left[v^{*} \boldsymbol{v}_{0}\right]-\boldsymbol{v}^{*} \cdot \partial_{x} \boldsymbol{v}_{0}-\partial_{x} p^{*}-\theta^{*} \partial_{x} \theta_{0}-c \partial_{x} v^{*}+\operatorname{Pr} \nabla^{2} v^{*}, \\
\frac{\partial}{\partial s} w^{*}(x-\Phi, y,-\omega s)=\nabla \cdot\left[w^{*} \boldsymbol{v}_{0}\right]-\boldsymbol{v}^{*} \cdot \partial_{y} \boldsymbol{v}_{0}-\partial_{y} p^{*}-\theta^{*} \partial_{y} \theta_{0}+\theta^{*}-c \partial_{x} w^{*}+\operatorname{Pr} \nabla^{2} w^{*}, \\
\frac{\partial}{\partial s} \theta^{*}(x-\Phi, y,-\omega s)=\nabla \cdot\left[\theta^{*} \boldsymbol{v}_{0}\right]+\operatorname{Pr} \operatorname{Ra} w^{*}-c \partial_{x} \theta^{*}+\nabla^{2} \theta^{*},
\end{gathered}
$$

where we denoted $\boldsymbol{U}_{\mathrm{p}}^{*}$ as $\boldsymbol{u}^{*}$ [defined by Eq. (A2)] and dropped the subscript 1 for simplicity. From a viewpoint of numerical analysis, a set of Eqs. (A18), (A19), (A20), and (A21) are similar to a set of Eqs. (1), (2), (3), and (4). In particular, the incompressible condition is given by Eq. (A18). The numerical method for the adjoint system given by Eqs. (A18), (A19), (A20), and (A21) is described in Appendix C.

\section{APPENDIX B: NUMERICAL METHODS FOR FLUID EQUATIONS}

In this Appendix, we describe the numerical scheme for the two-dimensional incompressible Navier-Stokes flow system with the periodic boundary condition on $x$ and the Dirichlet zero boundary condition on $y$ under the Boussinesq approximation. Equations (2), (3), and (4) can also be represented as

$$
\begin{gathered}
\partial_{t} v=N_{v}-\partial_{x} p+\operatorname{Pr} \nabla^{2} v, \\
\partial_{t} w=N_{w}-\partial_{y} p+\operatorname{Pr} \operatorname{Ra} \theta+\operatorname{Pr} \nabla^{2} w, \\
\partial_{t} \theta=N_{\theta}+\nabla^{2} \theta,
\end{gathered}
$$

where

$$
\begin{gathered}
N_{v}=-v \partial_{x} v-w \partial_{y} v, \\
N_{w}=-v \partial_{x} w-w \partial_{y} w, \\
N_{\theta}=-v \partial_{x} \theta-w \partial_{y} \theta+w .
\end{gathered}
$$

As in Eqs. (8), (9), and (10), the boundary condition on $y$ is given by

$$
\left.v\right|_{y= \pm 1}=\left.w\right|_{y= \pm 1}=\left.\theta\right|_{y= \pm 1}=0 .
$$

Using the incompressible condition given by Eq. (1), we can obtain the Poisson equation for the pressure $p$ as

$$
\nabla^{2} p=\partial_{x} N_{v}+\partial_{y}\left(N_{w}+\operatorname{Pr} \operatorname{Ra} \theta\right) .
$$

Here, the boundary condition on $y$ for the pressure $p$ is given by

$$
\left.\partial_{y} p\right|_{y= \pm 1}=-\left.\operatorname{Pr} \partial_{x} \partial_{y} v\right|_{y= \pm 1} .
$$

As in Refs. [70-72], we apply an improved projection scheme as follows. First, the time integration of Eq. (B3) is obtained by the following equation:

$$
\begin{aligned}
& \frac{3 \theta(n+1)-4 \theta(n)+\theta(n-1)}{2 \Delta t} \\
& \quad=2 N_{\theta}(n)-N_{\theta}(n-1)+\nabla^{2} \theta(n+1),
\end{aligned}
$$

where the boundary condition on $y$ is given by

$$
\left.\theta(n+1)\right|_{y= \pm 1}=0 \text {. }
$$

Second, the predictor for the pressure is obtained by the following equation:

$$
\begin{aligned}
\nabla^{2} p(\star)= & +\partial_{x}\left[2 N_{v}(n)-N_{v}(n-1)\right]+\partial_{y}\left[2 N_{w}(n)\right. \\
& \left.-N_{w}(n-1)+\operatorname{Pr} \operatorname{Ra} \theta(n+1)\right],
\end{aligned}
$$

where the boundary condition on $y$ is given by

$$
\left.\partial_{y} p(\star)\right|_{y= \pm 1}=-\left.\operatorname{Pr}\left[2 \partial_{x} \partial_{y} v(n)-\partial_{x} \partial_{y} v(n-1)\right]\right|_{y= \pm 1} .
$$

Third, the predictor for the fluid velocity is obtained by the following equations:

$$
\begin{aligned}
& \frac{3 v(\star)-4 v(n)+v(n-1)}{2 \Delta t} \\
& =2 N_{v}(n)-N_{v}(n-1)-\partial_{x} p(\star)+\operatorname{Pr} \nabla^{2} v(\star), \\
& \frac{3 w(\star)-4 w(n)+w(n-1)}{2 \Delta t} \\
& =2 N_{w}(n)-N_{w}(n-1)-\partial_{y} p(\star)+\operatorname{Pr} \operatorname{Ra} \theta(n+1) \\
& \quad+\operatorname{Pr} \nabla^{2} w(\star),
\end{aligned}
$$

where the boundary condition on $y$ is given by

$$
\left.v(\star)\right|_{y= \pm 1}=\left.w(\star)\right|_{y= \pm 1}=0 .
$$

Fourth, the scalar function for the correction step is obtained by the following equation:

$$
\nabla^{2} \phi(\star)=\partial_{x} v(\star)+\partial_{y} w(\star),
$$

where the boundary condition on $y$ is given by

$$
\left.\partial_{y} \phi(\star)\right|_{y= \pm 1}=0 .
$$

Finally, the fluid velocity and pressure are corrected by the following equations:

$$
\begin{aligned}
& v(n+1)=v(\star)-\frac{\partial}{\partial x} \phi(\star), \\
& w(n+1)=w(\star)-\frac{\partial}{\partial y} \phi(\star), \\
& p(n+1)=p(\star)+\frac{3}{2 \Delta t} \phi(\star) .
\end{aligned}
$$

As a spatial approximation, we apply the Fourier spectral method for $x$ and Chebyshev spectral method for $y$. 


\section{APPENDIX C: NUMERICAL METHODS FOR ADJOINT EQUATIONS}

In this Appendix, we describe the numerical scheme for the adjoint system with the periodic boundary condition on $x$ and the Dirichlet zero boundary condition on $y$. The scheme is almost parallel to the method described in Appendix B for the fluid system. When the traveling velocity is zero $(c=0)$, Eqs. (A19), (A20), and (A21) can also be represented as

$$
\begin{gathered}
\partial_{s} v^{*}=N_{v}^{*}-\partial_{x} p^{*}-\theta^{*} \partial_{x} \theta_{0}+\operatorname{Pr} \nabla^{2} v^{*}, \\
\partial_{s} w^{*}=N_{w}^{*}-\partial_{y} p^{*}-\theta^{*} \partial_{y} \theta_{0}+\theta^{*}+\operatorname{Pr} \nabla^{2} w^{*}, \\
\partial_{s} \theta^{*}=N_{\theta}^{*}+\nabla^{2} \theta^{*},
\end{gathered}
$$

where

$$
\begin{gathered}
N_{v}^{*}=\partial_{x}\left[v^{*} v_{0}\right]+\partial_{y}\left[v^{*} w_{0}\right]-v^{*} \partial_{x} v_{0}-w^{*} \partial_{x} w_{0}, \\
N_{w}^{*}=\partial_{x}\left[w^{*} v_{0}\right]+\partial_{y}\left[w^{*} w_{0}\right]-v^{*} \partial_{y} v_{0}-w^{*} \partial_{y} w_{0}, \\
N_{\theta}^{*}=\partial_{x}\left[\theta^{*} v_{0}\right]+\partial_{y}\left[\theta^{*} w_{0}\right]+\operatorname{Pr} \operatorname{Ra} w^{*} .
\end{gathered}
$$

As in Eqs. (A11), (A12), and (A13), the adjoint boundary condition on $y$ is

$$
\left.v^{*}\right|_{y= \pm 1}=\left.w^{*}\right|_{y= \pm 1}=\left.\theta^{*}\right|_{y= \pm 1}=0 .
$$

Using the incompressible condition given by Eq. (A18), we can obtain the Poisson equation for the adjoint pressure $p^{*}$ as

$$
\nabla^{2} p^{*}=\partial_{x}\left(N_{v}^{*}-\theta^{*} \partial_{x} \theta_{0}\right)+\partial_{y}\left(N_{w}^{*}-\theta^{*} \partial_{y} \theta_{0}+\theta^{*}\right) .
$$

Here, the boundary condition on $y$ for the adjoint pressure $p^{*}$ is given by

$$
\left.\partial_{y} p^{*}\right|_{y= \pm 1}=-\left.\operatorname{Pr} \partial_{x} \partial_{y} v^{*}\right|_{y= \pm 1} .
$$

As in Appendix B, we apply an improved projection scheme as follows. First, the time integration of Eq. (C3) is obtained by the following equation:

$$
\begin{aligned}
& \frac{3 \theta^{*}(n+1)-4 \theta^{*}(n)+\theta^{*}(n-1)}{2 \Delta s} \\
& \quad=2 N_{\theta}^{*}(n)-N_{\theta}^{*}(n-1)+\nabla^{2} \theta^{*}(n+1),
\end{aligned}
$$

where the boundary condition on $y$ is given by

$$
\left.\theta^{*}(n+1)\right|_{y= \pm 1}=0 .
$$

Second, the predictor for the adjoint pressure is obtained by the following equation:

$$
\begin{aligned}
\nabla^{2} p^{*}(\star)= & +\partial_{x}\left[2 N_{v}^{*}(n)-N_{v}^{*}(n-1)-\theta^{*}(n+1) \partial_{x} \theta_{0}(n+1)\right] \\
& +\partial_{y}\left[2 N_{w}^{*}(n)-N_{w}^{*}(n-1)\right. \\
& \left.-\theta^{*}(n+1) \partial_{y} \theta_{0}(n+1)+\theta^{*}(n+1)\right], \quad(\mathrm{C} 12)
\end{aligned}
$$

where the boundary condition on $y$ is given by

$$
\left.\partial_{y} p^{*}(\star)\right|_{y= \pm 1}=-\left.\operatorname{Pr}\left[2 \partial_{x} \partial_{y} v^{*}(n)-\partial_{x} \partial_{y} v^{*}(n-1)\right]\right|_{y= \pm 1} .
$$

Third, the predictor for the adjoint fluid velocity is obtained by the following equations:

$$
\begin{aligned}
& \frac{3 v^{*}(\star)-4 v^{*}(n)+v^{*}(n-1)}{2 \Delta s} \\
& =2 N_{v}^{*}(n)-N_{v}^{*}(n-1)-\partial_{x} p^{*}(\star) \\
& \quad-\theta^{*}(n+1) \partial_{x} \theta_{0}(n+1)+\operatorname{Pr} \nabla^{2} v^{*}(\star), \quad(\mathrm{C} 14) \\
& \frac{3 w^{*}(\star)-4 w^{*}(n)+w^{*}(n-1)}{2 \Delta s} \\
& =2 N_{w}^{*}(n)-N_{w}^{*}(n-1)-\partial_{y} p^{*}(\star)-\theta^{*}(n+1) \partial_{y} \theta_{0}(n+1) \\
& +\theta^{*}(n+1)+\operatorname{Pr} \nabla^{2} w^{*}(\star),
\end{aligned}
$$

where the boundary condition on $y$ is given by

$$
\left.v^{*}(\star)\right|_{y= \pm 1}=\left.w^{*}(\star)\right|_{y= \pm 1}=0 .
$$

Fourth, the scalar function for the correction step is obtained by the following equation:

$$
\nabla^{2} \phi^{*}(\star)=\partial_{x} v^{*}(\star)+\partial_{y} w^{*}(\star),
$$

where the boundary condition on $y$ is given by

$$
\left.\partial_{y} \phi^{*}(\star)\right|_{y= \pm 1}=0 \text {. }
$$

Finally, the adjoint fluid velocity and adjoint pressure are corrected by the following equations:

$$
\begin{aligned}
& v^{*}(n+1)=v^{*}(\star)-\frac{\partial}{\partial x} \phi^{*}(\star), \\
& w^{*}(n+1)=w^{*}(\star)-\frac{\partial}{\partial y} \phi^{*}(\star), \\
& p^{*}(n+1)=p^{*}(\star)+\frac{3}{2 \Delta s} \phi^{*}(\star) .
\end{aligned}
$$

As a spatial approximation, we apply the Fourier spectral method for $x$ and Chebyshev spectral method for $y$.
[1] A. T. Winfree, The Geometry of Biological Time, 2nd ed. (Springer, New York, 2001).

[2] Y. Kuramoto, Chemical Oscillations, Waves, and Turbulence (Dover, New York, 2003).

[3] A. Pikovsky, M. Rosenblum, and J. Kurths, Synchronization: A Universal Concept in Nonlinear Sciences (Cambridge University Press, Cambridge, UK, 2001).

[4] S. C. Manrubia, A. S. Mikhailov, and D. H. Zanette, Emergence of Dynamical Order: Synchronization Phenomena in Complex Systems (World Scientific, Singapore, 2004).
[5] G. V. Osipov, J. Kurths, and C. Zhou, Synchronization in Oscillatory Networks (Springer, New York, 2007).

[6] F. C. Hoppensteadt and E. M. Izhikevich, Weakly Connected Neural Networks (Springer, New York, 1997).

[7] E. M. Izhikevich, Dynamical Systems in Neuroscience: The Geometry of Excitability and Bursting (MIT Press, Cambridge, MA, 2007).

[8] G. B. Ermentrout and D. H. Terman, Mathematical Foundations of Neuroscience (Springer, New York, 2010).

[9] G. B. Ermentrout, Type I membranes, phase resetting curves, and synchrony, Neural Comput. 8, 979 (1996). 
[10] E. Brown, J. Moehlis, and P. Holmes, On the phase reduction and response dynamics of neural oscillator populations, Neural Comput. 16, 673 (2004).

[11] H. Nakao, Phase reduction approach to synchronization of nonlinear oscillators, Contemp. Phys. 57, 188 (2016).

[12] B. Pietras and A. Daffertshofer, Network dynamics of coupled oscillators and phase reduction techniques, Phys. Rep. 819, 1 (2019).

[13] S. H. Strogatz, From Kuramoto to Crawford: Exploring the onset of synchronization in populations of coupled oscillators, Phys. D (Amsterdam, Neth.) 143, 1 (2000).

[14] J. A. Acebrón, L. L. Bonilla, C. J. Pérez Vicente, F. Ritort, and R. Spigler, The Kuramoto model: A simple paradigm for synchronization phenomena, Rev. Mod. Phys. 77, 137 (2005).

[15] A. Arenas, A. Díaz-Guilera, J. Kurths, Y. Moreno, and C. Zhou, Synchronization in complex networks, Phys. Rep. 469, 93 (2008).

[16] A. Pikovsky and M. Rosenblum, Dynamics of globally coupled oscillators: Progress and perspectives, Chaos 25, 097616 (2015).

[17] F. A. Rodrigues, T. K. DM. Peron, P. Ji, and J. Kurths, The Kuramoto model in complex networks, Phys. Rep. 610, 1 (2016).

[18] P. Ashwin, S. Coombes, and R. Nicks, Mathematical frameworks for oscillatory network dynamics in neuroscience, J. Math. Neurosci. 6, 2 (2016).

[19] T. Stankovski, T. Pereira, P. V. E. McClintock, and A. Stefanovska, Coupling functions: Universal insights into dynamical interaction mechanisms, Rev. Mod. Phys. 89, 045001 (2017).

[20] Y. Kawamura, H. Nakao, and Y. Kuramoto, Collective phase description of globally coupled excitable elements, Phys. Rev. E 84, 046211 (2011).

[21] Y. Kawamura, Collective phase reduction of globally coupled noisy dynamical elements, Phys. Rev. E 95, 032225 (2017).

[22] Y. Kawamura and H. Nakao, Collective phase description of oscillatory convection, Chaos 23, 043129 (2013).

[23] Y. Kawamura and H. Nakao, Noise-induced synchronization of oscillatory convection and its optimization, Phys. Rev. E 89, 012912 (2014).

[24] H. Nakao, T. Yanagita, and Y. Kawamura, Phase-Reduction Approach to Synchronization of Spatiotemporal Rhythms in Reaction-Diffusion Systems, Phys. Rev. X 4, 021032 (2014).

[25] Y. Kawamura, S. Shirasaka, T. Yanagita, and H. Nakao, Optimizing mutual synchronization of rhythmic spatiotemporal patterns in reaction-diffusion systems, Phys. Rev. E 96, 012224 (2017).

[26] Y. Kawamura and R. Tsubaki, Phase Reduction Approach to Elastohydrodynamic Synchronization of Beating Flagella, Phys. Rev. E 97, 022212 (2018).

[27] P. Manneville, Dissipative Structures and Weak Turbulence (Academic Press, New York, 1990).

[28] H. Mori and Y. Kuramoto, Dissipative Structures and Chaos (Springer, New York, 1997).

[29] M. C. Cross and H. Greenside, Pattern Formation and Dynamics in Nonequilibrium Systems (Cambridge University Press, Cambridge, UK, 2009).

[30] M. C. Cross and P. C. Hohenberg, Pattern formation outside of equilibrium, Rev. Mod. Phys. 65, 851 (1993).
[31] A. S. Mikhailov and K. Showalter, Control of waves, patterns, and turbulence in chemical systems, Phys. Rep. 425, 79 (2006).

[32] I. R. Epstein, I. B. Berenstein, M. Dolnik, V. K. Vanag, L. Yang, and A. M. Zhabotinsky, Coupled and forced patterns in reaction-diffusion systems, Philos. Trans. R. Soc. A 366, 397 (2008).

[33] K. Showalter and I. R. Epstein, From chemical systems to systems chemistry: Patterns in space and time, Chaos 25, 097613 (2015).

[34] A. S. Mikhailov and G. Ertl (eds.), Engineering of Chemical Complexity (World Scientific, Singapore, 2013).

[35] A. S. Mikhailov and G. Ertl (eds.), Engineering of Chemical Complexity II (World Scientific, Singapore, 2014).

[36] M. Ghil and S. Childress, Topics in Geophysical Fluid Dynamics: Atmospheric Dynamics, Dynamo Theory, and Climate Dynamics (Springer, New York, 1987).

[37] B. M. Boubnov and G. S. Golitsyn, Convection in Rotating Fluids (Kluwer Academic, London, 1995).

[38] S. Chandrasekhar, Hydrodynamic and Hydromagnetic Stability (Dover, New York, 1981).

[39] M. Lappa, Thermal Convection: Patterns, Evolution, and Stability (Wiley, Chichester, UK, 2010).

[40] M. Lappa, Rotating Thermal Flows in Natural and Industrial Processes (Wiley, Chichester, UK, 2012).

[41] M. Ghil, P. Read, and L. Smith, Geophysical flows as dynamical systems: The influence of Hide's experiments, Astron. Geophys. 51, 4.28 (2010).

[42] P. L. Read, M. J. Bel, D. W. Johnson, and R. M. Small, Quasiperiodic and chaotic flow regimes in a thermally driven, rotating fluid annulus, J. Fluid Mech. 238, 599 (1992).

[43] F. J. R. Eccles, P. L. Read, A. A. Castrejón-Pita, and T. W. N. Haine, Synchronization of modulated traveling baroclinic waves in a periodically forced, rotating fluid annulus, Phys. Rev. E 79, 015202(R) (2009).

[44] A. A. Castrejón-Pita and P. L. Read, Synchronization in a Pair of Thermally Coupled Rotating Baroclinic Annuli: Understanding Atmospheric Teleconnections in the Laboratory, Phys. Rev. Lett. 104, 204501 (2010).

[45] P. L. Read, X. Morice-Atkinson, E. J. Allen, and A. A. Castrejón-Pita, Phase synchronization of baroclinic waves in a differentially heated rotating annulus experiment subject to periodic forcing with a variable duty cycle, Chaos $\mathbf{2 7}, 127001$ (2017).

[46] Y. Kawamura and H. Nakao, Phase description of oscillatory convection with a spatially translational mode, Phys. D (Amsterdam, Neth.) 295-296, 11 (2015).

[47] U. M. Ascher and L. R. Petzold, Computer Methods for Ordinary Differential Equations and Differential-Algebraic Equations (SIAM, Philadelphia, 1998).

[48] P. Lin, A sequential regularization method for time-dependent incompressible Navier-Stokes equations, SIAM J. Numer. Anal. 34, 1051 (1997).

[49] E. M. Izhikevich, Weakly connected quasi-periodic oscillators, FM interactions, and multiplexing in the brain, SIAM J. Appl. Math. 59, 2193 (1999).

[50] K. Taira and H. Nakao, Phase-response analysis of synchronization for periodic flows, J. Fluid Mech. 846, R2 (2018).

[51] M. Iima, Jacobian-free algorithm to calculate the phase sensitivity function in the phase reduction theory and its applications to Kármán’s vortex street, Phys. Rev. E 99, 062203 (2019). 
[52] R. E. Goldstein, E. Lauga, A. I. Pesci, and M. R. E. Proctor, Elastohydrodynamic synchronization of adjacent beating flagella, Phys. Rev. Fluids 1, 073201 (2016).

[53] G. De Canio, E. Lauga, and R. E. Goldstein, Spontaneous oscillations of elastic filaments induced by molecular motors, J. R. Soc. Interface 14, 20170491 (2017).

[54] Y. Kawamura, H. Nakao, and Y. Kuramoto, Noise-induced turbulence in nonlocally coupled oscillators, Phys. Rev. E 75, 036209 (2007).

[55] Y. Kawamura, H. Nakao, K. Arai, H. Kori, and Y. Kuramoto, Collective Phase Sensitivity, Phys. Rev. Lett. 101, 024101 (2008).

[56] Y. Kawamura, H. Nakao, K. Arai, H. Kori, and Y. Kuramoto, Phase synchronization between collective rhythms of globally coupled oscillator groups: Noisy identical case, Chaos 20, 043109 (2010).

[57] Y. Kawamura, Collective phase dynamics of globally coupled oscillators: Noise-induced anti-phase synchronization, Phys. D (Amsterdam, Neth.) 270, 20 (2014).

[58] M. C. Cross, Phase dynamics of convective rolls, Phys. Rev. A 27, 490 (1983).

[59] M. C. Cross and A. C. Newell, Convection patterns in large aspect ratio systems, Phys. D (Amsterdam, Neth.) 10, 299 (1984).

[60] H. R. Brand and M. C. Cross, Phase dynamics for the wavy vortex state of the Taylor instability, Phys. Rev. A 27, 1237 (1983).

[61] H. R. Brand, Nonlinear phasedynamics for the spatially periodic states of the Taylor instability, Prog. Theor. Phys. 71, 1096 (1984).

[62] P. Coullet and S. Fauve, Propagative Phase Dynamics for Systems with Galilean Invariance, Phys. Rev. Lett. 55, 2857 (1985).

[63] S. Fauve, E. W. Bolton, and M. E. Brachet, Nonlinear oscillatory convection: A quantitative phase dynamics approach, Phys. D (Amsterdam, Neth.) 29, 202 (1987).

[64] Y. Kuramoto, Instability and turbulence of wavefronts in reaction-diffusion systems, Prog. Theor. Phys. 63, 1885 (1980).

[65] Y. Kuramoto, Phase dynamics of weakly unstable periodic structures, Prog. Theor. Phys. 71, 1182 (1984).

[66] Y. Kuramoto, On the reduction of evolution equations in extended systems: The underlying universal structure, Prog. Theor. Phys. Suppl. 99, 244 (1989).

[67] T. Watanabe, M. Iima, and Y. Nishiura, Spontaneous formation of traveling localized structures and their asymptotic behavior in binary fluid convection, J. Fluid Mech. 712, 219 (2012).

[68] P. Hignett, A. A. White, R. D. Carter, W. D. N. Jackson, and R. M. Small, A comparison of laboratory measurements and numerical simulations of baroclinic wave flows in a rotating cylindrical annulus, Q. J. R. Meteorol. Soc. 111, 131 (1985).

[69] A. Randriamampianina, W.-G. Früh, P. L. Read, and P. Maubert, Direct numerical simulations of bifurcations in an air-filled rotating baroclinic annulus, J. Fluid Mech. 561, 359 (2006).

[70] R. Peyret, Spectral Methods for Incompressible Viscous Flow (Springer, New York, 2002).

[71] G. E. Karniadakis, M. Israeli, and S. A. Orszag, High-order splitting methods for the incompressible Navier-Stokes equations, J. Comput. Phys. 97, 414 (1991).

[72] S. Hugues and A. Randriamampianina, An improved projection scheme applied to pseudospectral methods for the incompressible Navier-Stokes equations, Int. J. Numer. Meth. Fluids 28, 501 (1998).

[73] I. Raspo, S. Hugues, E. Serre, A. Randriamampianina, and P. Bontoux, A spectral projection method for the simulation of complex three-dimensional rotating flows, Comp. Fluids 31, 745 (2002)

[74] I. Mercader, O. Batiste, and A. Alonso, An efficient spectral code for incompressible flows in cylindrical geometries, Comp. Fluids 39, 215 (2010).

[75] A. Hagberg and E. Meron, Pattern formation in non-gradient reaction-diffusion systems: The effects of front bifurcations, Nonlinearity 7, 805 (1994).

[76] M. Yadome, K. Ueda, and M. Nagayama, Chaotic motion of propagating pulses in the Gray-Scott model, Phys. Rev. E 83, 056207 (2011).

[77] P. L. Read and A. A. Castrejón-Pita, Phase synchronization between stratospheric and tropospheric quasi-biennial and semiannual oscillations, Q. J. R. Meteorol. Soc. 138, 1338 (2012).

[78] K. Rajendran, I. M. Moroz, P. L. Read, and S. M. Osprey, Synchronization of the equatorial QBO by the annual cycle in tropical upwelling in a warming climate, Q. J. R. Meteorol. Soc. 142, 1111 (2016).

[79] D. Zwillinger, Handbook of Differential Equations, 3rd ed. (Academic Press, New York, 1998).

[80] J. P. Keener, Principles of Applied Mathematics: Transformation and Approximation, 2nd ed. (Perseus, Cambridge, MA, 2000).

[81] L. C. Evans, Partial Differential Equations, 2nd ed. (American Mathematical Society, Providence, RI, 2010). 